Article

\title{
Exploiting the Freshwater Shrimp Neocaridina denticulata as Aquatic Invertebrate Model to Evaluate Nontargeted Pesticide Induced Toxicity by Investigating Physiologic and Biochemical Parameters
}

\author{
Petrus Siregar ${ }^{1,2}$, Michael Edbert Suryanto ${ }^{2}$, Kelvin H.-C. Chen ${ }^{3}{ }^{\mathbb{D}}$, Jong-Chin Huang ${ }^{3} \mathbb{D}$, Hong-Ming Chen ${ }^{4}$, \\ Kevin Adi Kurnia ${ }^{2}$, Fiorency Santoso ${ }^{2}$, Akhlaq Hussain ${ }^{2}$, Bui Thi Ngoc Hieu 1,2 , Ferry Saputra ${ }^{2}$, \\ Gilbert Audira ${ }^{1,2}$, Marri Jmelou M. Roldan ${ }^{5}$ (D), Rey Arturo Fernandez ${ }^{6}$, Allan Patrick G. Macabeo ${ }^{6, *(D)}$, \\ Hong-Thih Lai ${ }^{4, *}$ and Chung-Der Hsiao ${ }^{1,2,7, * \text { (D) }}$
}

check for updates

Citation: Siregar, P.; Suryanto, M.E.; Chen, K.H.-C.; Huang, J.-C.; Chen, H.-M.; Kurnia, K.A.; Santoso, F.; Hussain, A.; Ngoc Hieu, B.T.; Saputra, F.; et al. Exploiting the Freshwater Shrimp Neocaridina denticulata as Aquatic Invertebrate Model to Evaluate Nontargeted Pesticide Induced Toxicity by Investigating Physiologic and Biochemical Parameters. Antioxidants 2021, 10, 391. https://doi.org/10.3390/ antiox10030391

Academic Editor: Victoria Cachofeiro

Received: 27 January 2021

Accepted: 2 March 2021

Published: 5 March 2021

Publisher's Note: MDPI stays neutral with regard to jurisdictional claims in published maps and institutional affiliations.

Copyright: (c) 2021 by the authors. Licensee MDPI, Basel, Switzerland. This article is an open access article distributed under the terms and conditions of the Creative Commons Attribution (CC BY) license (https:// creativecommons.org/licenses/by/ $4.0 /)$.
1 Department of Chemistry, Chung Yuan Christian University, Chung-Li 320314, Taiwan; g10765013@cycu.edu.tw (P.S.); hieubtn90@gmail.com (B.T.N.H.); g10701304@cycu.edu.tw (G.A.)

2 Department of Bioscience Technology, Chung Yuan Christian University, Chung-Li 320314, Taiwan; g10865014@cycu.edu.tw (M.E.S.); g10865016@cycu.edu.tw (K.A.K.); g10766011@cycu.edu.tw (F.S.); g10765017@cycu.edu.tw (A.H.); g10865013@cycu.edu.tw (F.S.)

3 Department of Applied Chemistry, National Pingtung University, Pingtung 900391, Taiwan; kelvin@mail.nptu.edu.tw (K.H.-C.C.); hjc@mail.nptu.edu.tw (J.-C.H.)

4 Department of Aquatic Biosciences, National Chiayi University, 300 University Rd., Chiayi 60004, Taiwan; wwwtt333@gmail.com

5 Faculty of Pharmacy and The Graduate School, University of Santo Tomas, Manila 1008, Philippines; mmroldan@ust.edu.ph

6 Laboratory for Organic Reactivity, Discovery and Synthesis (LORDS), Research Center for the Natural and Applied Sciences, University of Santo Tomas, Manila 1008, Philippines; reyarturo.tapia.fernandez@gmail.com

7 Center for Nanotechnology, Chung Yuan Christian University, Chung-Li 320314, Taiwan

* Correspondence: agmacabeo@ust.edu.ph (A.P.G.M.); htlai@mail.ncyu.edu.tw (H.-T.L.); cdhsiao@cycu.edu.tw (C.-D.H.); Tel.: +886-32653545 (C.-D.H.); Fax: +886-32653599 (C.-D.H.)

\begin{abstract}
As a nicotinoid neurotoxic insecticide, imidacloprid (IMI) works by disrupting nerve transmission via nicotinic acetylcholine receptor (nAChR). Although IMI is specifically targeting insects, nontarget animals such as the freshwater shrimp, Neocaridina denticulata, could also be affected, thus causing adverse effects on the aquatic environment. To investigate IMI toxicity on nontarget organisms like N. denticulata, their physiology (locomotor activity, heartbeat, and gill ventilation) and biochemical factors (oxidative stress, energy metabolism) after IMI exposure were examined. IMI exposure at various concentrations $(0.03125,0.0625,0.125,0.25,0.5$, and $1 \mathrm{ppm})$ to shrimp after 24 , $48,72 \mathrm{~h}$ led to dramatic reduction of locomotor activity even at low concentrations. Meanwhile, IMI exposure after $92 \mathrm{~h}$ caused reduced heartbeat and gill ventilation at high concentrations. Biochemical assays were performed to investigate oxidative stress and energy metabolism. Interestingly, locomotion immobilization and cardiac activity were rescued after acetylcholine administration. Through molecular docking, IMI demonstrated high binding affinity to nAChR. Thus, locomotor activity and heartbeat in shrimp after IMI exposure may be caused by nAChR blockade and not alterations caused by oxidative stress and energy metabolism. To summarize, $N$. denticulata serves as an excellent and sensitive aquatic invertebrate model to conduct pesticide toxicity assays that encompass physiologic and biochemical examinations.
\end{abstract}

Keywords: imidacloprid; insecticide; Neocaridina denticulata; shrimp; locomotion activity; cardiac activity; ecotoxicity

\section{Introduction}

Pesticides are a class of heterogeneous chemicals with significant public health benefits by increasing food production and decreasing food-borne and vector-borne diseases. 
However, studies have shown their adverse effects on nontarget organisms, including humans depending on the agent and the exposure [1]. Epidemiological studies suggest that exposure to pesticides may increase the incidence of respiratory diseases [2], neurologic dysfunctions [3], carcinogenicity [4], and reproductive disorders [5]. More than 20,000 pesticide products with nearly 900 active ingredients are registered as insecticides, miticides, herbicides, rodenticides, nematicides, and fungicides. Insecticides are considered the most useful pesticides among chemical or biological agents that control diseases caused by insect pests.

Imidacloprid [1-(6-chloro-3-pyridylmethyl)-2-nitroiminoimidazolidine] (IMI) belongs to a class of synthetic insecticides called neonicotinoids and is used widely to control insect pests on crops and fleas on domestic animals [6]. Contemporary insecticide products, like neonicotinoids, are often detected in aquatic systems [7]. Neonicotinoids potentially damage biological systems and antioxidant defenses. Exposure of susceptible organisms may lead to overstimulation in the nervous system, impairment of physiology, and death [8]. IMI is taken up by insects through contact and/or ingestion and binds to the nicotinic acetylcholine receptor (nAChR), allowing disruption of nerve impulses. It is selective to insect $n A C h R$ receptors and has much less pronounced effects in mammals $[9,10]$. The receptor anchors are ligand-gated ion channels and are involved in synaptic transmissions in the central nervous system [11]. There is evidence that IMI interaction with the $\alpha$ subunit of nAChRs contributes to its partial agonist actions and its selectivity for insect $\mathrm{nAChRs} \mathrm{[12].} \mathrm{Sales} \mathrm{of} \mathrm{neonicotinoids} \mathrm{are} \mathrm{becoming} \mathrm{fast-growing} \mathrm{globally,} \mathrm{brought} \mathrm{about}$ by low selectivity to insects and apparent safety for mammals $[13,14]$. Since IMI is a known substitute to other commercial pesticides, relative risks, and benefits associated with it must be compared to other pesticides.

Crustaceans such as the freshwater shrimp Neocaridina denticulata are used as animal models for screening toxicity because they exhibit rapid and sensitive responses to toxic substances. Additional characteristics of $N$. denticulata, include having small organismal size $(2-3 \mathrm{~cm})$, short reproduction period (4-5 months), and high larval production rate (50-100 individuals/breed) as beneficial aquatic indicators in assessing environmental pollution. N. denticulata has been used to determine pollutant effects, such as chlordane and lindane, on growth and reproductive hormones [15]. In 2005, the Environmental Protection Administration of Taiwan indicated its use during the conduct of acute toxicity assays to evaluate the safety levels of bodies of water or effluents coming from industrial districts [16].

Nontarget aquatic organisms can be exposed to IMI from spray drift, leaching, or run-off in agricultural contexts. Upon entry of IMI into aquatic environments, its dissipation may be aggravated by photolysis. While the possibility of neonicotinoid pesticide exposure exists in both targeted and nontargeted animals, there is limited data on studies illustrating IMI toxicity or adverse effects on nontarget aquatic organisms, particularly efforts describing effects on behavioral and biochemical alterations. Thus, we examined IMI's possible acute effects on complex behavioral activity and neurotoxicity using biochemical, molecular biology, coupled with computational techniques such as molecular docking in the shrimp, $N$. denticulata, as an aquatic animal model.

It is noteworthy to mention that IMI acts by binding to nicotinic acetylcholine receptors and consequently disrupts nervous system functions [17]. It binds to nAChR in insect nervous tissues via neurotransmission dysregulation at cholinergic synapsis, thus causing death $[18,19]$. IMI selectively binds to $\mathrm{NAChR}[20]$ and prevents acetylcholine from binding to $\mathrm{nAChR}$, causing blockage of regulated receptor binding functions of $\mathrm{nAChR}[21,22]$. Acetylcholine is a neurotransmitter in the nervous system [23], particularly consumed in the neuromuscular junction and motor neurons to activate the muscles $[23,24]$. As a working hypothesis, we speculated that IMI might block nAChR binding of acetylcholine by selectively acting on the nicotinic acetylcholine receptors. In this study, we also illustrated that IMI affects both shrimp locomotion and cardiac activities. We also investigated the effects of exposing $N$. denticulata to acetylcholine, a biochemical that binds to $\mathrm{nAChR}$, to 
check rescuing properties after IMI treatment. We thought that acetylcholine might increase locomotion and cardiac activity by saturating $\mathrm{nAChR}$ before binding to IMI.

\section{Materials and Methods}

\subsection{Overview of Experimental Design to Conduct Acute Toxicity Test in Shrimp}

Lack of specificity usually becomes the main problem of insecticides, thus, affecting both invertebrates and vertebrates other than insects in general [25]. The freshwater shrimp $N$. denticulata was used as a model in this study to represent aquatic invertebrates affected by insecticide carried away by overflow from terrestrial to receiving aquatic environments. As such, shrimp have been used as a model and bioindicator in assessing chemical contaminations in the environment due to high sensitivity [26].

The acute toxicity test in shrimp was conducted in six concentrations $(0.03125,0.0625$, $0.125,0.25,0.5$, and $1 \mathrm{ppm}$ ) of IMI from low to high concentrations, and a control. These six concentrations were chosen based on $\mathrm{LC}_{50}$ and $\mathrm{EC}_{50}$ data based on a previous procedure reported by our laboratory (see Sections 2.3 and 2.4, respectively). Twelve shrimp were exposed in each group during the first experiment. The toxicity test (locomotor activity and heartbeat measurement) results were monitored at 24, 48, 72, and $96 \mathrm{~h}$ exposure to IMI. After $24 \mathrm{~h}$ of exposure to IMI, the treated shrimp's locomotor activity was recorded using the ZebraBox instrument (Viewpoint Co., Lyon France). The locomotor activity of treated shrimp was observed every day until $72 \mathrm{~h}$ (day 3) post-exposure (Figure 1, red arrows) was reached. In addition to locomotor activity, heartbeat and gill ventilation (based on the maxilliped movement) were also measured to validate endpoints of the most sensitive markers for reporting IMI toxicity. The shrimp's heart rate and gill ventilation were examined during $96 \mathrm{~h}$ exposure to IMI (Figure 1, blue arrows). After which, the remaining shrimp were frozen and sacrificed for biochemical testing using enzyme-linked immunoassay (ELISA) screens (Figure 1, green arrow). The EC $_{50}$ dose of IMI was assessed after $96 \mathrm{~h}$ of IMI exposure (Figure 1). Complete immobilization occurred in shrimp after receiving higher concentrations ( 0.5 and $1 \mathrm{ppm}$ ) of IMI treatment. The $\mathrm{EC}_{50}$ was estimated at $0.51 \mathrm{ppm}$ for IMI on inhibiting locomotor activity. Furthermore, to check whether acetylcholine could rescue IMI adverse effects in shrimp, a similar experimental design was used along with the addition of acetylcholine into treated shrimp. After IMI treatment for $24 \mathrm{~h}$, treated shrimp were moved from IMI water, cleansed, and exposed to acetylcholine. Rescued shrimp locomotor and heartbeat were observed every day until day 3 (Figure 1). The concentrations used for the rescue experiment were 0.1 and $1 \mathrm{ppm}$ of IMI along with acetylcholine 0.1 and $1 \mathrm{ppm}$. The differences between the first experiment (IMI toxicity test) and the rescue experiment are the total $\mathrm{n}$ number and the addition of acetylcholine in the rescue experiment. In the IMI toxicity test, the total n number for locomotor activity and heartbeat measurement were 12 shrimp for each concentration group, which is treatment $(0.03125,0.0625,0.0125,0.25,0.5$, and $1 \mathrm{ppm})$ and for control. Meanwhile, for the rescue experiment the total $\mathrm{n}$ number is 24 shrimp for each group, which is the treatment group ( 0.5 and $1 \mathrm{ppm})$ and the control group.

\subsection{Animal Housing}

$N$. denticulate with an average total length of $1-1.5 \mathrm{~cm}$ for experimental assays were provided by the Freshwater Resource Center of National Chiayi University, Chiayi City, Taiwan. Shrimp were stocked for 7 days at $26.5^{\circ} \mathrm{C}$ in a recirculating system water under a 10/14 dark/light cycle to confirm healthy conditions before the assay. Circulating water in the aquarium was filtered by reverse osmosis at $\mathrm{pH}$ 7.0-7.5. Shrimp were fed twice a day (morning and evening) for 7 days prior to the conduct of the assay. All procedures in this study were approved by the Animal Ethics Committee of the Chung Yuan Christian University (Approval ID 107030). 


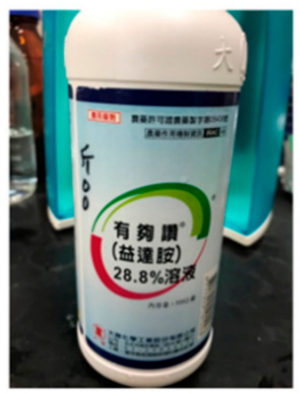

\section{Imidacloprid}<smiles>O=[N+]([O-])/N=C1\NCCN1Cc1ccc(Cl)nc1</smiles>

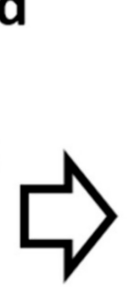

\section{Neocaridina denticulata}

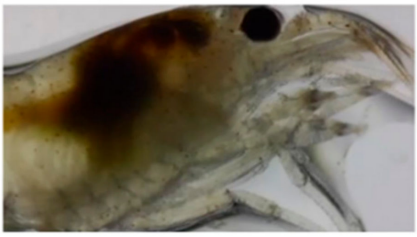

Acute toxicity experiment

96hrs EC 50
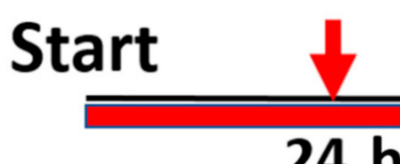

$24 \mathrm{~h}$

Start

Acetylcholine rescue experiment

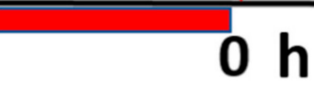

$0 \mathrm{~h} \quad 24 \mathrm{~h}$
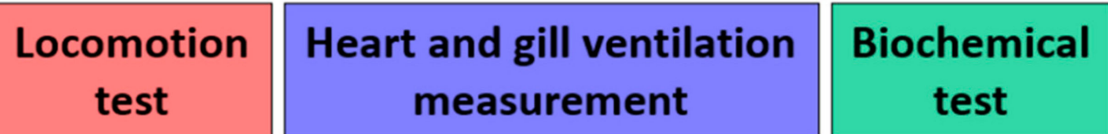

\section{Molecular docking}

Figure 1. Overview of experimental design to conduct imidacloprid (IMI) acute toxicity and acetylcholine rescue test in shrimp (Neocaridina denticulata). For the acute toxicity test, IMI was exposed to N. denticulata for $96 \mathrm{~h}$. By 24, 48 , and $72 \mathrm{~h}$ post-exposure, the locomotory activity changes of IMI-treated shrimp were measured. After $96 \mathrm{~h}$, the heartbeat and gill ventilation (measured by maxilliped beating rate) of IMI-treated shrimp were measured. The experimental animals were subjected to enzyme-linked immunosorbent assay (ELISA) assay for biomarker expression measurements. For the rescue experiment, shrimp were pretreated with $1 \mathrm{ppm}$ IMI for $24 \mathrm{~h}$ and transferred to either clear water or acetylcholine solution. After $0,24,48$, and $72 \mathrm{~h}$ post-exposure, the rescued shrimp's locomotory activity changes were measured and compared.

\subsection{Imidacloprid Treatment}

Imidacloprid was obtained commercially as a $28.8 \%$ solution (Great Victory Chemical Industry Co. Ltd., Yunlin County, Taiwan). Its $\mathrm{LC}_{50}$ was reported as $0.91 \mu \mathrm{g} / \mathrm{L}$ in aquatic invertebrate [10]. IMI toxicity assays were initiated by diluting $28.8 \%$ stock solutions with water to working concentrations of $0.03125,0.0625,0.125,0.25,0.5$, and 1 ppm. Exposure studies were conducted in 4 days in plastic tanks containing 20 shrimp with a dimension of $12 \mathrm{~cm}(\mathrm{~L}) \times 9 \mathrm{~cm}(\mathrm{~W}) \times 5 \mathrm{~cm}(\mathrm{H})$ filled with $200 \mathrm{~mL}$ working solution for each treatment.

\section{4. $E C_{50}$ Measurement for Immobilization}

Concentrations at $50 \%$ of maximal effect $\left(\mathrm{EC}_{50}\right)$ test for locomotion impairment were determined using six different concentrations of IMI of $0.03125,0.0625,0.125,0.25,0.5$, and $1 \mathrm{ppm}$. Shrimp were incubated with different IMI concentrations, and the corresponding locomotor activities were measured every day after $24,48,72$, and $96 \mathrm{~h}$ of exposure. $\mathrm{EC}_{50}$ curves for locomotion impairment at 24, 48, 72, and $96 \mathrm{~h}$ were constructed based on the immobilization of the shrimp from each test group.

\subsection{Locomotion Tracking and Quantification}

After IMI incubation, individual shrimp were placed into wells of a 6-well transparent plate (one shrimp per well/per concentration); each well with $\approx 10 \mathrm{~mL}$ of $0.03125,0.0625$, $0.125,0.25,0.5$, or $1 \mathrm{ppm}$ IMI) in two replicates. For video recording, each plate was placed into Zebrabox instrument (Viewpoint Co., Lyon, France) attached with a remote 
recording device equipped with a camera and infrared light-emitting where light can be controlled. The ViewPoint system, a video tracking software (Viewpoint Co., France), was set in tracking mode to record individual shrimp activity. Shrimp were initially habituated for $20 \mathrm{~min}$, followed by $20 \mathrm{~min}$ of recording in the light cycle condition. After which, the total distance swam for each shrimp was calculated. Swimming speed thresholds were set and used to define three different speed thresholds as (1) bursting $(>5.0 \mathrm{~cm} / \mathrm{s})$, is a short, intermittent, and powerful bout of activity; (2) cruising $(1.0 \leq \mathrm{s} \leq 5.0 \mathrm{~cm} / \mathrm{s})$, which captures commonly measured shrimp speeds; (3) freezing $(<1.0 \mathrm{~cm} / \mathrm{s})$ will display the minimal activity of shrimp. All results were binned in 1-min intervals, resulting in 20 data points.

\subsection{Heartbeat and Gill Ventilation (Based on Maxilliped Movement) Measurement}

Heartbeat and gill ventilation (based on maxilliped movement) measurements were performed based on our previously established ImageJ-based dynamic pixel change method [27]. The video was captured using a digital charge-coupled device (CCD) (SK2700HDMI-T2, Zgenebio, Taipei, Taiwan) mounted on a dissecting microscope with a frame rate setting at 60 frames per second (fps). In this experiment, 12 shrimp were exposed to IMI. Each treated shrimp was recorded using CCD camera. N. denticulata after IMI exposure were used to conduct heart rate and gill ventilation assay using ImageJ as a major platform. A region of interest (ROI) was selected using a circle tool, and the selected ROI was targeted on either whole heart or maxilliped regions. Stack Difference and Time Series Analyzer Plugin were used to detect dynamic pixel changes over time. Graphic of beat rhythm was then performed using Origin 9.1 software (Originlab Corporation, Northampton, MA, USA). Peak times were calculated using the Peak Analyzer function in Origin 9.1 and processed using Microsoft Excel (Microsoft 2016 version, Seattle, WA, USA) to obtain time interval and beats per minute (bpm) data. Beats per minute were obtained by multiplying the time interval (in seconds) between two peaks by a factor of 60 .

\subsection{ELISA for Measuring Biomarker Expression}

After completion of heartbeat and gill ventilation measurements, shrimp were sacrificed from both control and treatment groups to conduct the biochemical assays. Shrimp whole-body was homogenized in ice in volumes of $50(\mathrm{v} / w)$ phosphate-buffered saline (PBS) at pH 7.2 using a bullet blender tissue homogenizer (Next Advance, Inc., Troy, NY, USA). The samples were then centrifuged at $12,000 \mathrm{rpm}$ for $20 \mathrm{~min}$ at $4{ }^{\circ} \mathrm{C}$. The supernatant was collected and stored at $-80^{\circ} \mathrm{C}$ for total protein estimation and ELISA tests. BCA Protein Assay Kit (23225, Thermo Fisher Scientific, Waltham, MA, USA) was used to measure total protein concentration. In quantifying biomarkers expression, six ELISA kits were used to measure reactive oxygen species (ROS) related to oxidative stress, lipid peroxidation markers of 4-hydroxynonenal (4-HNE), and thiobarbituric acid reactive substances (TBARS) to determine lipid peroxidation in tissue samples. Additionally, energy metabolism bioassays for measuring glucose, pyruvate, and lactate levels were carried out. All commercial ELISA kits were purchased from Zgenebio Inc. (Taipei, Taiwan), and tests were conducted based on the manufacturer's instructions. Color absorbance was analyzed at $450 \mathrm{~nm}$ wavelength using a microplate reader (Multiskan GO, Thermo Fisher Scientific, Waltham, MA, USA).

\subsection{In Silico Methods}

\subsubsection{Protein Preparation}

The protein crystal structure of the Acetylcholine Binding Protein (Ls-AChBP, PDB ID: 2ZJU) from the great pond snail (Lymnaea stagnalis) was obtained from the Protein Data Bank (https:/ / www.rcsb.org/, accessed on 1 February 2021) and its 3D structure (Figure A1) was incorporated into the UCSF chimera platform [28]. It was prepared initially by removing all nonstandard residues. Chains $A$ and $B$ were left in preparation for the docking to represent a subunit interface. The Acetylcholine Binding Protein structure was minimized by the steepest descent method (100 steps-step size $0.02 \AA$ ) and conjugate gradient method (10 steps-step size $0.02 \AA$ ). 


\subsubsection{Ligand Preparation}

Imidacloprid and acetylcholine were used as ligands for the docking experiments. The structures were then converted from SMILES format to SYBYL mol2 file using Avogadro (version 1.2.0), an open-source molecular builder, and were added to the UCSF Chimera platform for docking [29].

\subsubsection{Molecular Docking}

UCSF Chimera was used as the platform for molecular docking studies [29]. The Gasteiger charge method computed using Amber's Antechamber module added the missing hydrogen atoms and appropriate charges in the docking preparation [30]. The docking procedure employed flexible ligands into flexible active site protocol, allowing the ligand's translational and rotational walk within the grid box [31]. Virtual screening of the prepared library was performed following the Broyden-Fletcher-Goldfarb-Shanoo (BFGS) algorithm of AutoDock Vina (version 1.5.6) [32]. Finally, the binding affinity was determined using UCSF Chimera and was visualized and analyzed through Biovia Discovery Studios (version 4.1).

\subsection{Acetylcholine Rescue Experiment}

Based on our results, IMI exposure induced a sharp immobilization in shrimp. An experiment was conducted to rescue the locomotor activity reduction in shrimp by adding acetylcholine to the IMI pretreated shrimp. Four test groups with 24 shrimp each were prepared and kept in $500 \mathrm{~mL}$ water tanks. The first group served as a control without any treatment. The other three groups were treated with $1 \mathrm{ppm}$ IMI for $24 \mathrm{~h}$. After $24 \mathrm{~h}$, two groups for rescue treatment were then transferred to acetylcholine solution, one in a low concentration $(0.1 \mathrm{ppm})$ and the other in a high concentration $(1 \mathrm{ppm})$, while the remaining group was transferred to normal water without acetylcholine treatment. The shrimp locomotor activity, heartbeat, and gill ventilation rate from each group were recorded at 0 , 24,48 , and $72 \mathrm{~h}$ post-exposure. For video recording, individual shrimp were placed into wells of a 6-well transparent plate (one shrimp per well/per concentration) and recorded for $20 \mathrm{~min}$ in three replicates. UMATracker, an open-source quantitative analysis for animal tracking [33], was used to track the shrimp's locomotion activity. The video was loaded to UMATracker, and by using a filter generator, the video background was computed to retrieve and distinguish animal images. Animal images were converted to grayscale and binarized by adjusting the threshold value. By thresholding the image binarization algorithm, we obtained the output result of the converted video frame. At the tracking step, we set the number of animals in the video frame and ran the tracking algorithm to detect each animal's position. After successfully tracking based on whole video frames, the data was saved as csv files format. These data contain XY coordinates and animals' position per frame, which were processed using Microsoft Excel. The total distance activity from all groups was measured and compared. For heartbeat and gill ventilation measurement, the process has been explained above.

\subsection{Biostatistics}

The experimental values between control and IMI treated groups were compared. For heartbeat and gill ventilation rate, the statistical tests were analyzed by a two-way ANOVA test continued with Dunnet's multiple comparisons test as the follow-up test. Rescue and acetylcholine exposure experiment data were expressed as percentages, with control day 0 as the comparison. For locomotor activity, time chronology assay, the statistical tests were conducted through the two-way ANOVA with the Geisser-Greenhouse correction with either Dunnett's multiple comparison test or the Kruskal-Wallis test with Dunn's multiple comparisons test as a follow-up test. For locomotor activity, most data were expressed as mean with SEM. We did not use median because several behavioral data have 0 value of median. In addition, if we used mean $+/-95 \%$ CI, some error bars will be less than 0 . 
Therefore, mean \pm SEM was reported to avoid confusion. Statistical tests were performed using GraphPad Prism (https:/ / www.graphpad.com/, accessed on 1 February 2021).

\section{Results}

\subsection{Imidacloprid Exposure Impaired Locomotor Activity in Shrimp}

The quantitative comparison of different doses of IMI toxicity on the shrimp locomotor activity is shown in Figure 2. The total distances were calculated on the first 24, 48, and $72 \mathrm{~h}$ prior to IMI exposure. Based on the results, significant reductions in total locomotor activity were observed in all IMI-treated shrimp. This alteration was visible immediately after 1-day IMI exposure, supported by a significant decrease in the shrimp total distance traveled observed during the assay, including the lowest concentration group $(0.03125 \mathrm{ppm})$ (Figure 2A,D). Furthermore, the statistically significant gaps in the shrimp total distance traveled between the control, and the treatment groups became more pronounced due to increased exposure concentrations and time (Figure 2B,C,E,F). At high concentration (1 ppm), shrimp locomotor activity consistently exhibited the lowest value compared to the other treated groups, starting from day one to day three of the test. The locomotor activity compromised after 3-day IMI exposure also can be found in Video S1. From locomotor activity trajectory (Figure 2G), it will be more straightforward to see the shrimp treated with IMI showed sharp locomotor activity decline compared to the control group. Even the lowest concentration $(0.03125 \mathrm{ppm})$ already exhibited stagnant locomotor activity (Figure 2A-G). Additionally, the total distance traveled in the control group was relatively high during the first day, while it was slightly reduced in the following days, probably due to food fasting. Overall, the results showed that IMI could significantly reduce shrimp locomotor activity based on factors such as treatment exposure, time, and dose.

\subsection{Imidacloprid Exposure Impaired Heartbeat and Gill Ventilation in Shrimp}

The effects of IMI exposure to heartbeat and gill ventilation are shown in Figure 3. To the best of our knowledge, we demonstrate for the first time the effect of pesticides on heartbeat and gill ventilation in $N$. denticulata. The heart is a vital organ for cardiovascular circulation in shrimp. Cardiovascular dysfunction has been used to evaluate environmental toxicants as significant risk factors in crustaceans [34,35]. The maxilliped is one of the three pairs of appendages located immediately behind the maxillae of shrimp and plays multiple functions on feeding and facilitating oxygen uptake [36,37]. Thus, heartbeat and maxilliped movement were selected as two additional endpoints for pesticide toxicity evaluation. The regions of interest (ROIs) in the heart and maxilliped for $N$. denticulata were highlighted in red and blue colors, respectively (Figure 3A).

In this study, shrimp exposed to IMI were not fed for the entire duration of the experiment. After shrimp exposure to IMI, the heartbeat and maxilliped movements were reduced compared to the control shrimp. At $96 \mathrm{~h}$ post-exposure, heartbeat rates of 0.5 and $1 \mathrm{ppm}$ IMI treatments were significantly decreased (Figure 3C), while heartbeat intervals of 0.5 and 1 ppm IMI were increased (Figure 3D) compared to the control. On the other hand, a reduction in maxilliped movement was also observed in shrimp exposed to $0.25,0.5$, and 1 ppm IMI (Figure 3E,F). Moreover, heartbeat and gill ventilation movement in free-feeding shrimp not exposed to IMI as control were also examined. Interestingly, shrimp with sufficient nutrition had a higher heartbeat and maxilliped movement when fasted groups were compared (Figure 3C-F). The heartbeat rates of shrimp with sufficient food, lack of nutrition, and $1 \mathrm{ppm}$ of IMI exposure were $194 \pm 21.13,135.2 \pm 5.5,86.56 \pm 15.62 \mathrm{bpm}$, respectively. On the other hand, the gill ventilation movement in shrimp with sufficient food, lack of nutrition, and 1 ppm of IMI exposure were $404.5 \pm 52.34,207.3 \pm 23.81$, $119.9 \pm 22.29 \mathrm{bpm}$, respectively. This result suggests feed conditions should be carefully controlled with consistency levels for shrimp heartbeat and maxilliped movement experiments to minimize potential variations. 
(A)
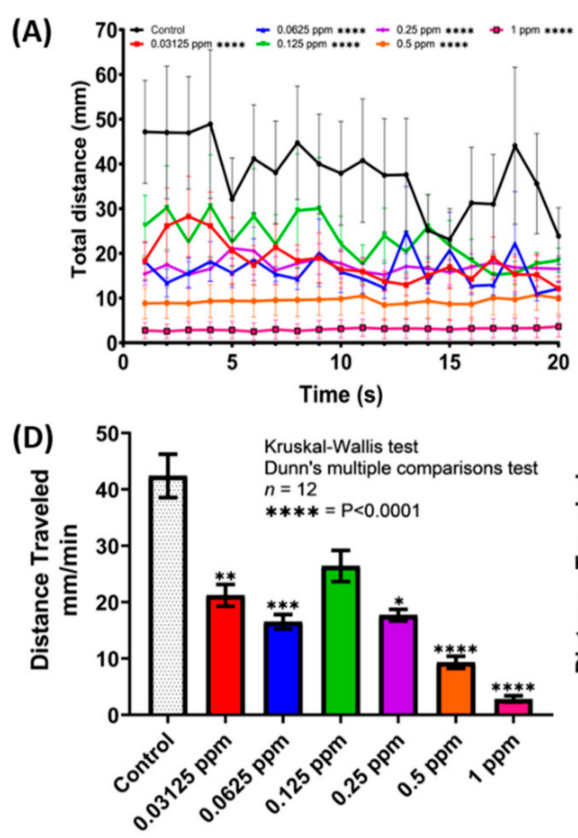

(B)
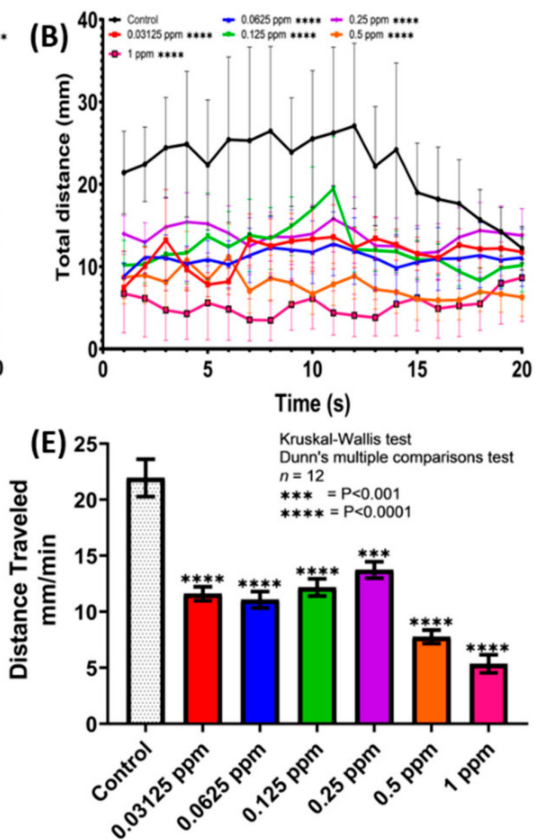
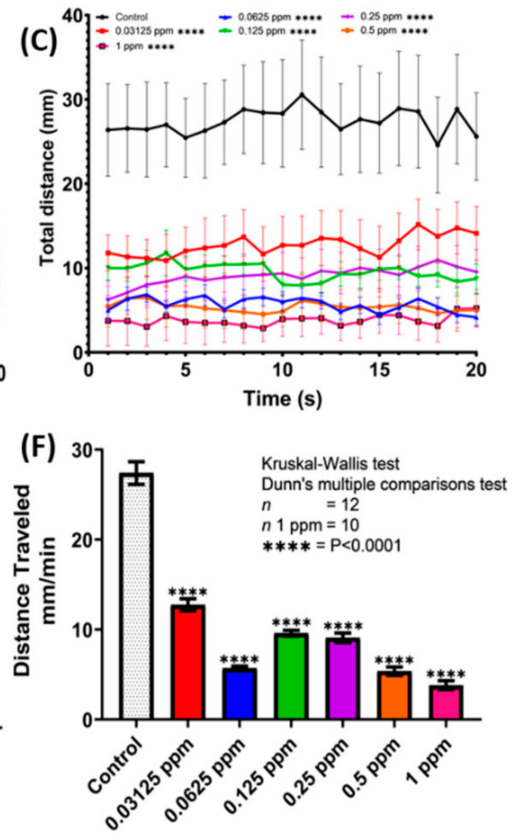

(G)

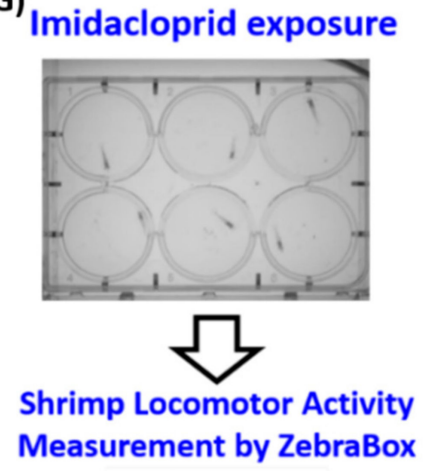

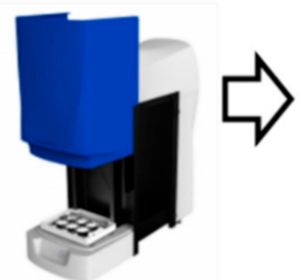

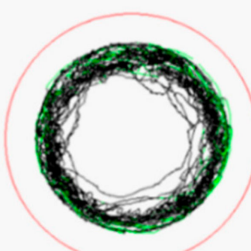

CONTROL
Total distance traveled within $\mathbf{2 0}$ min
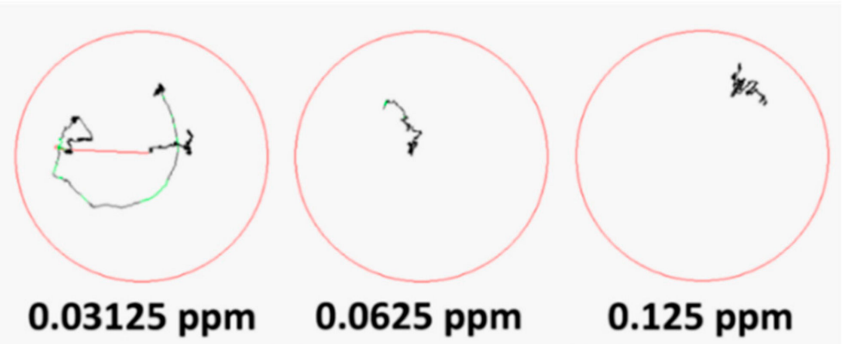

$0.125 \mathrm{ppm}$
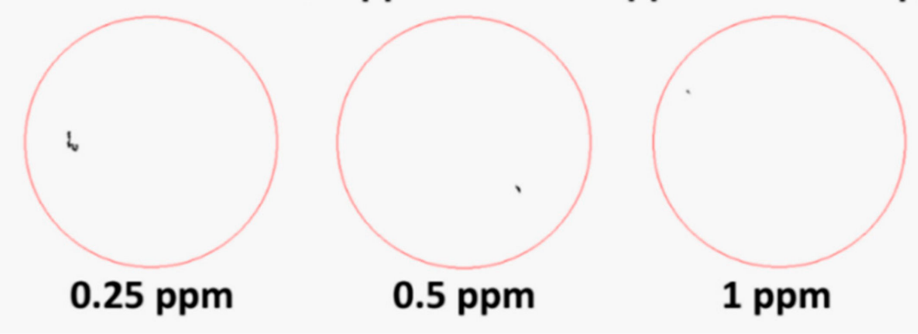

Figure 2. (A-C) Average distance traveled per minute by untreated and imidacloprid (IMI) treated shrimp (Neocaridina denticulata) after 24, 48, $72 \mathrm{~h}$ exposure, respectively, in given concentrations (0.03125 ppm (red), $0.0625 \mathrm{ppm}$ (blue), $0.125 \mathrm{ppm}$ (green), $0.25 \mathrm{ppm}$ (purple), $0.5 \mathrm{ppm}$ (orange), $1 \mathrm{ppm}$ (pink)). The data are expressed as the mean with SEM and were analyzed by two-way ANOVA with the Geisser-Greenhouse correction. Dunnett's multiple comparison test for comparing all treatments with control was carried out to observe the main column (IMI) effect. (D-F) Total average distance traveled per minute for $20 \mathrm{~min}$ locomotor activity test by the control (untreated), and IMI treated shrimp after 24, 48, 72 h exposure. (G) The trajectory of shrimp treated with imidacloprid within 20 min video recording by Zebrabox instrument. The data are expressed as mean with SEM and were analyzed by the Kruskal-Wallis test continued with Dunn's multiple comparisons test as a follow-up test $\left(n=12\right.$ for all of the groups, except 1 ppm group on the $72 \mathrm{~h}$ exposure time interval $(n=10)\left({ }^{*} p<0.05\right.$; $\left.{ }^{* *} p<0.01 ;{ }^{* * *} p<0.001 ;{ }^{* * * *} p<0.0001\right)$. 


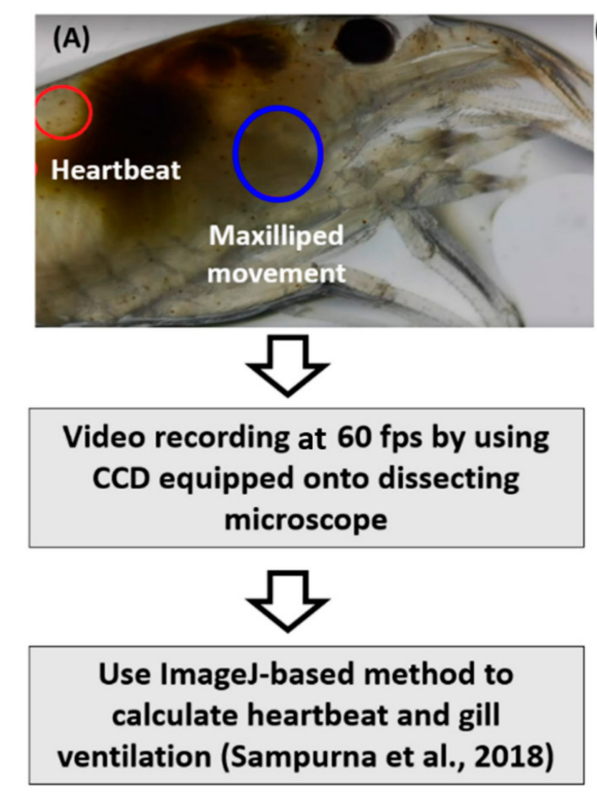

(B) Heartbeat (No Starvation)

$194 \pm 21.13 \mathrm{bpm}$

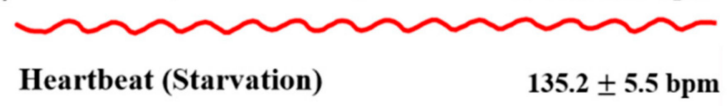

nNNvNN

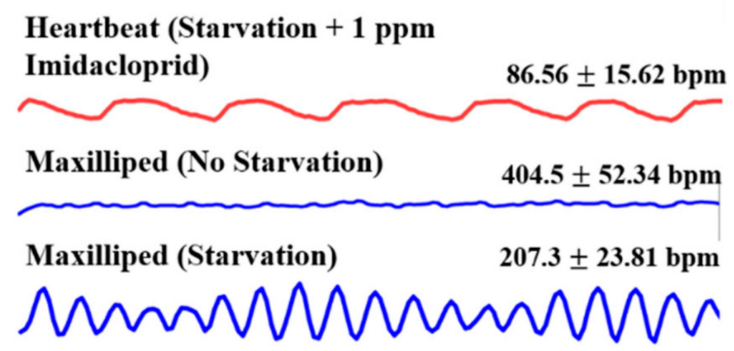

Maxilliped (Starvation + 1 ppm Imidacloprid)
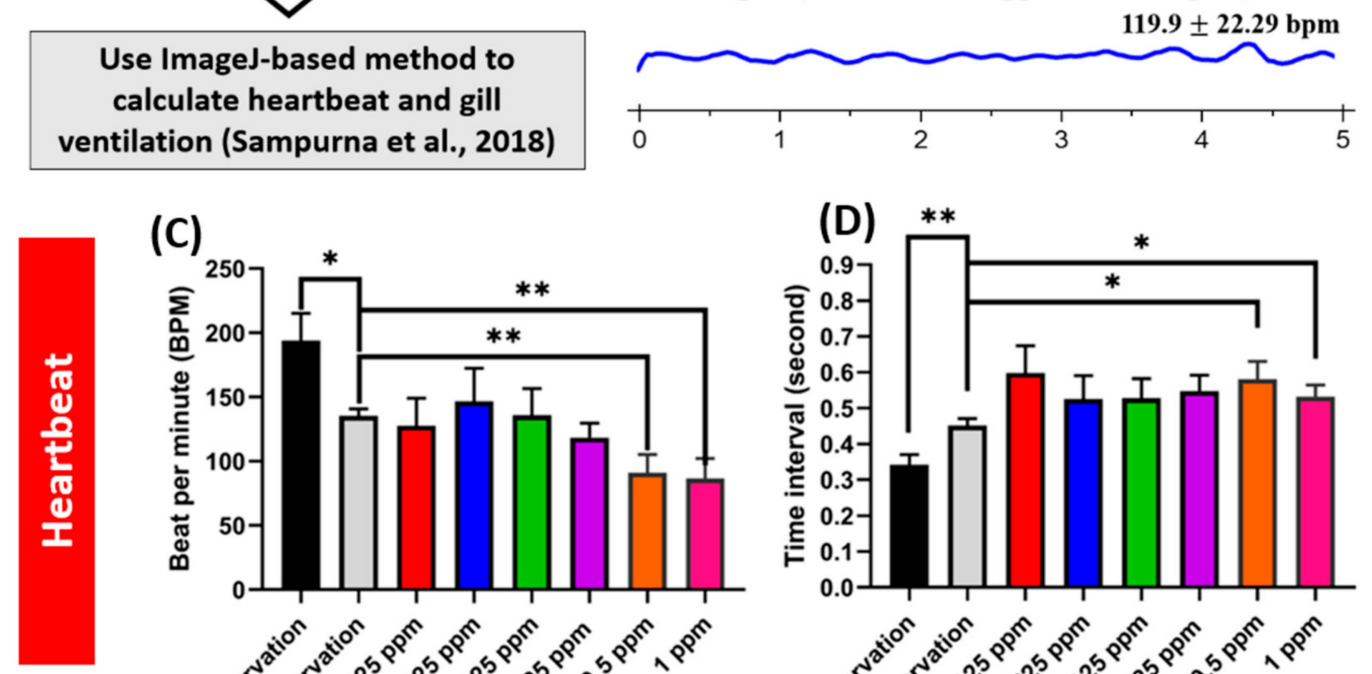

(C)
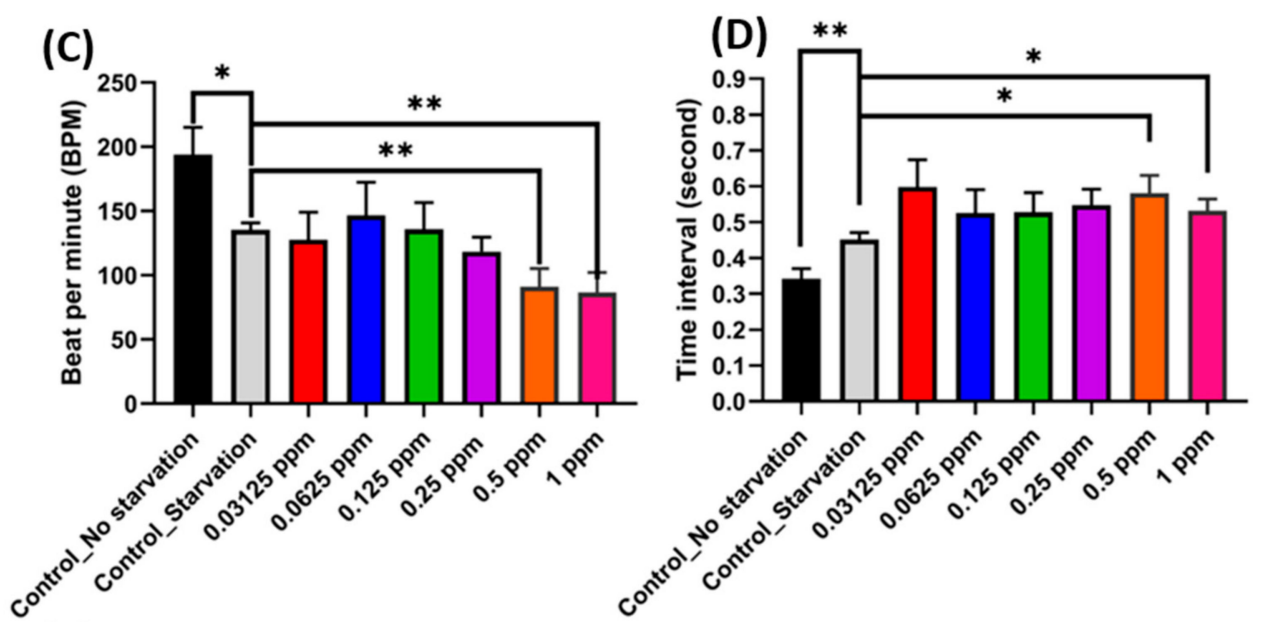

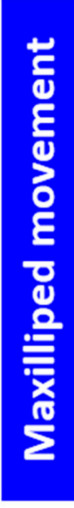

(E)

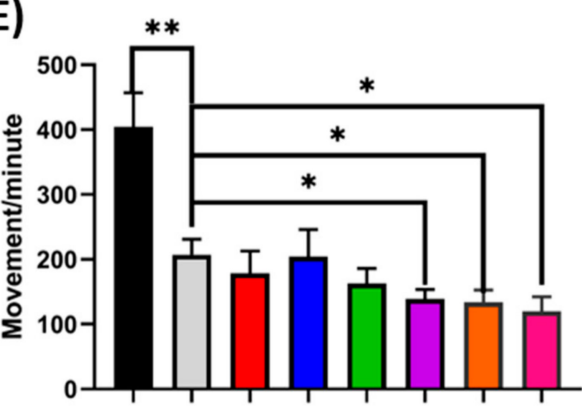

(F)

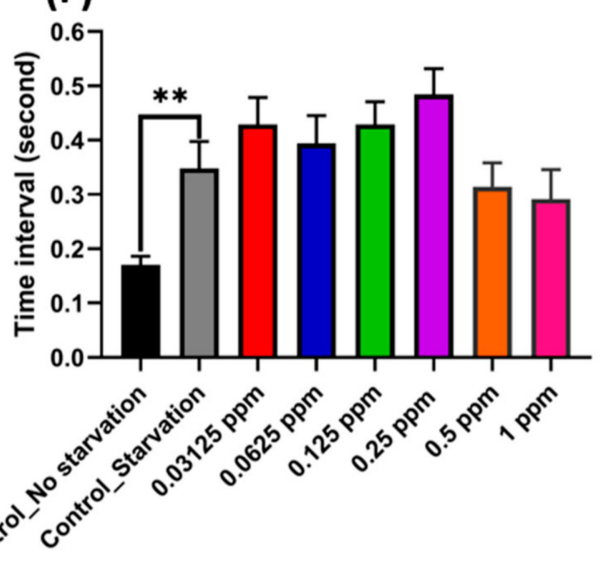

Figure 3. The heartbeat and gill ventilation (based on maxilliped beating) in shrimp (Neocaridina denticulata) are affected by starvation and pesticide pollution. (A) Overview of the method used to measure heartbeat and maxilliped beating in the shrimp (left panel). (B) The dynamic pixel changes over time for the heart (red color) or maxilliped (blue color) with no starvation, starvation, or imidacloprid (IMI) treatments. Evaluation of IMI toxicity at different doses by monitoring (C) heart rate, (D) heartbeat interval, (E) gill ventilation rate, and (F) gill ventilation interval in N. denticulata. The data are expressed as mean with SEM and were analyzed using two-way ANOVA along with Dunnet's multiple comparisons as a follow-up test. $\left({ }^{*} p<0.05 ;{ }^{* *} p<0.01\right)$. 
In addition, we also extracted heartbeat and gill ventilation chronological patterns using the ImageJ software (Figure 3B). The dynamic pixel intensity is higher when the heart is pumping, and a higher peak rhythm is formed, while lower peak rhythm forms are noted when there is heart relaxation. Heartbeat and gill ventilation were calculated by dividing $1 \mathrm{~min}(60 \mathrm{~s})$ with a time interval. A faster heartbeat will form additional peaks that will lead to a shortened time interval between two successive peaks. A slower heartbeat will form a longer time interval. In Figure 3B (red panel), the peak loosens as the heartbeat slows in shrimp treated with IMI. Similar results were noted in gill ventilation movement (Figure 3B blue panel). The heartbeat and gill ventilation move for control and IMI exposed shrimp can be found in Videos S2 and S3, respectively.

Further evaluation of heartbeat and gill ventilation movement alteration were checked using the Poincare plot. The heartbeat and maxilliped movement were observed in the following groups: no starvation (control), starvation, and starvation with $1 \mathrm{ppm}$ (IMI treatment). The Poincaré plot is a scattergram generated by plotting each R-R interval towards the previous one, based on our previous methods [38]. R-R interval is the interval between successive beats defined by heartbeats and gill ventilation. An increased and extensive scatter region visualized high variability. With the Poincare plot, the irregularity can be analyzed quantitatively based on standard deviations (sd1 and sd2). The sd1 and sd2 are two descriptors of R-R intervals in the Poincaré plot determined by fitting an ellipse to the plotted shape. Sd1 is the standard deviation perpendicular to the line-ofidentity, while sd2 is the standard deviation along the line-of-identity $[39,40]$. A higher value of sd1 and sd2 indicates high variability changes in heart rate and gill ventilation. Variability of heart rate in starvation $(\mathrm{sd} 1=0.0314$; $\mathrm{sd} 2=0.0243$ ) (Figure $4 \mathrm{~B})$ and IMI treatment $(\mathrm{sd} 1=0.1122 ; \mathrm{sd} 2=0.0471)$ (Figure $4 \mathrm{C}$ ) were higher compared to the control group $(\mathrm{sd} 1=0.0179 ; \mathrm{sd} 2=0.0158)($ Figure $4 \mathrm{~A})$. Meanwhile, highest variability of gill ventilation was displayed in the control group ( $\mathrm{sd} 1=0.0906$; $\mathrm{sd} 2=0.0697$ ) (Figure 4D), followed by the IMI treatment $(\mathrm{sd} 1=0.0703 ; \mathrm{sd} 2=0.0547)($ Figure $4 \mathrm{~F})$, and the starvation group with the least variability $(\mathrm{sd} 1=0.0192 ; \mathrm{sd} 2=0.0136)$ (Figure $4 \mathrm{E})$ for gill ventilation movement. This result revealed that IMI treatment could cause irregularity in $N$. denticulata heartbeats and gill ventilation.

\subsection{Biomarker Expression in Shrimp after Imidacloprid Administration}

A biochemical assay was performed to identify the possible mechanism of locomotion immobilization, cardiac and gill ventilation alterations. For this purpose, we analyzed several essential biomarkers related to oxidative stress of reactive oxygen species (ROS), 4-hydroxynonenal (4-HNE), and thiobarbituric acid reactive substances (TBARS) to measure the extent of DNA damage after IMI exposure to $N$. denticulata. Pesticide-induced oxidative stress is mediated by excessive ROS production [41]. A recent study on Drosophila melanogaster indicated chronic IMI exposure could elevate the ROS level and damage the fly's vision [42]. However, in this acute toxicity test for N. denticulata, we did not find any significant ROS level alteration after the short-term exposure to IMI (Figure 5A). The ROS level was found slightly higher at a concentration of $0.5 \mathrm{ppm}$ but did not reach a significant difference with the control and other treatment groups. Oxidative stress also induces lipid peroxidation in cellular membranes and forms 4-HNE and TBARS as by-products. 4-HNE and TBARS test was used to quantify lipid peroxidation caused by oxidative stress in the biological system $[43,44]$. Similar to ROS results, there are no significant differences in 4-HNE and TBARS levels in all groups (Figure 5B,C). Energy metabolism of glucose, pyruvate, and lactate was also measured. No significant results for biomarkers in shrimp related to oxidative stress and energy metabolism for all tested concentrations were observed (Figure 5). We suggest that locomotion, heartbeat, and gill ventilation hypoactivity may not be associated with oxidative stress or alteration in energy metabolism. Hypoactivity could be caused by neurotransmitter disruption related to IMI's mechanism of action as an inhibitor of acetylcholinesterase. IMI binds to acetylcholine receptors preventing transmission of impulses, resulting in acetylcholine accumulation [45]. Therefore, we experimented 
to restore normal acetylcholine levels to further investigate whether acetylcholine rescue could restore locomotion, heartbeat, and gill ventilation hypoactivity in N. denticulata after treatment with IMI.
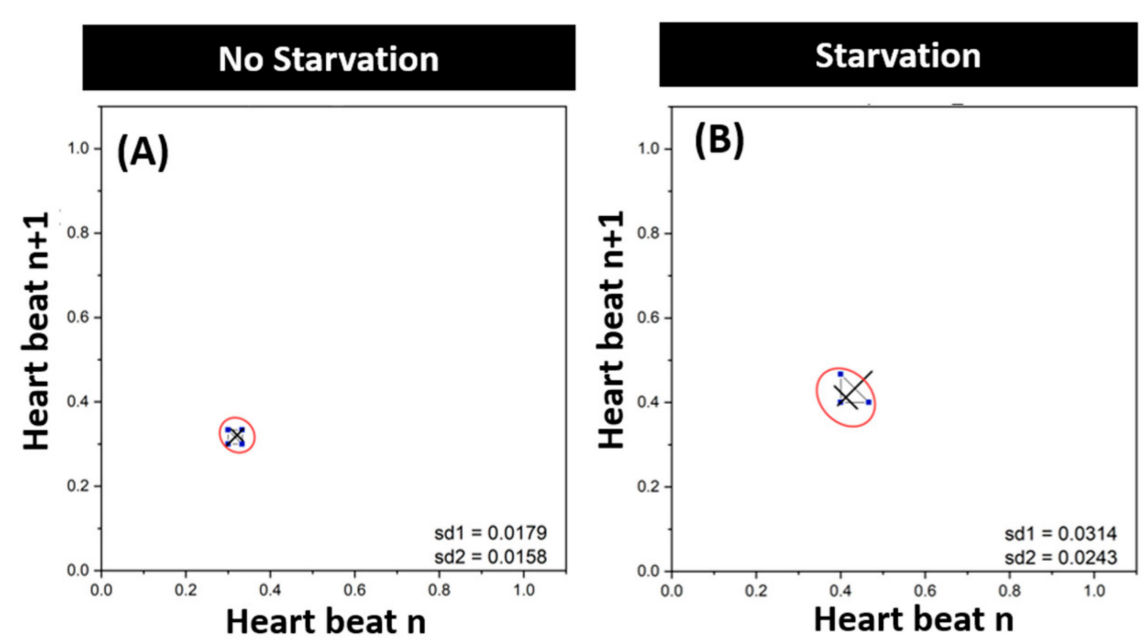

Starvation + 1 ppm Imidacloprid
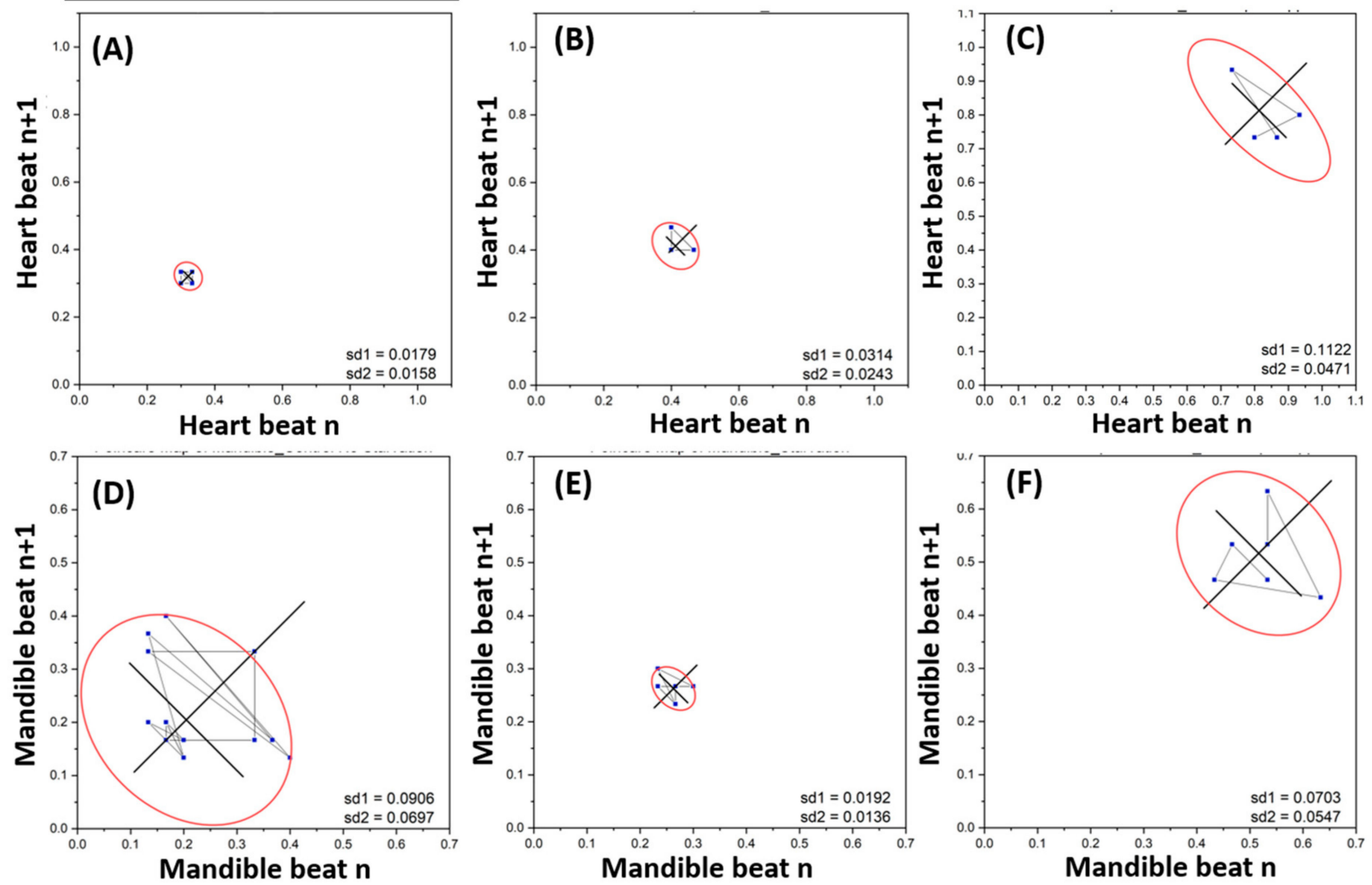

Figure 4. Evaluation of imidacloprid (IMI) toxicity at different doses by monitoring heartbeat and gill ventilation regularity. Poincaré plot showing the heartbeat (A-C) and maxilliped beating (D-F) regularity when treated with no starvation (A,D), starvation (B,E), or IMI (C,F) treatments. The data are expressed as mean with SEM, and the one-way ANOVA tested the significance with a nonparametric Mann-Whitney test.

\subsection{Locomotion Impairment Can Be Rescued by Acetylcholine Administration}

Nicotinic acetylcholine receptors (nAChRs) are ligand-gated ion channels that mediate fast cholinergic synaptic transmission in insects and vertebrate nervous systems [45,46]. The high affinity between IMI and nAChR led us to question whether overexposure to exogenous acetylcholine can compete with IMI for rescue. Thus, shrimp were initially incubated with $1 \mathrm{ppm}$ IMI for $24 \mathrm{~h}$ to immobilize their locomotor activity. The shrimp were then transferred to a new tank containing freshwater, $0.1 \mathrm{ppm}$ acetylcholine, or $1 \mathrm{ppm}$ acetylcholine. The locomotor activity was monitored at day 0 for IMI exposure and days 1,2 , and 3 after exposure to acetylcholine for rescue. As such, $1 \mathrm{ppm}$ IMI was found to sharply induce locomotor immobilization by exhibiting approximately 5 -fold decrement compared to the untreated control group (Figure 6A,B). This immobilization phenotype can sustain for at least 3 days even after the shrimp were transferred to clean water (Figure 6A). The total distance in the IMI group rescue with freshwater was significantly reduced in four consecutive days, with a $p$-value $<0.0001$. Interestingly, the compromised locomotor activity triggered after IMI treatment can be rescued by supplying $1 \mathrm{ppm}$ exogenous acetylcholine (Figure 6A). The rescue effect can be observed even after 3 days of acetylcholine exposure (Figure 6A,E,I). From days 0, 1, and 2 after the rescue, locomotion from two rescue treatments (acetylcholine 0.1 and $1 \mathrm{ppm}$ ) showed lower total distance 
traveled with significant difference ( $p$ value $<0.0001$ ) compared to the control. On the other hand, an increase in locomotor activity was observed in both rescue treatment groups on day 3. The $0.1 \mathrm{ppm}$ acetylcholine treatment showed significantly higher total distance traveled than the IMI group treated with freshwater (Figure 6A,E,I). However, locomotion activity from this group was significantly lower compared to the control group. The 1ppm acetylcholine group on day 3 displayed higher total distance traveled and displayed no significant difference than the control (Figure 6A). These results indicate that higher acetylcholine concentration with an equal balance to IMI (1:1 concentration) can restore shrimp locomotion activity.

(A)

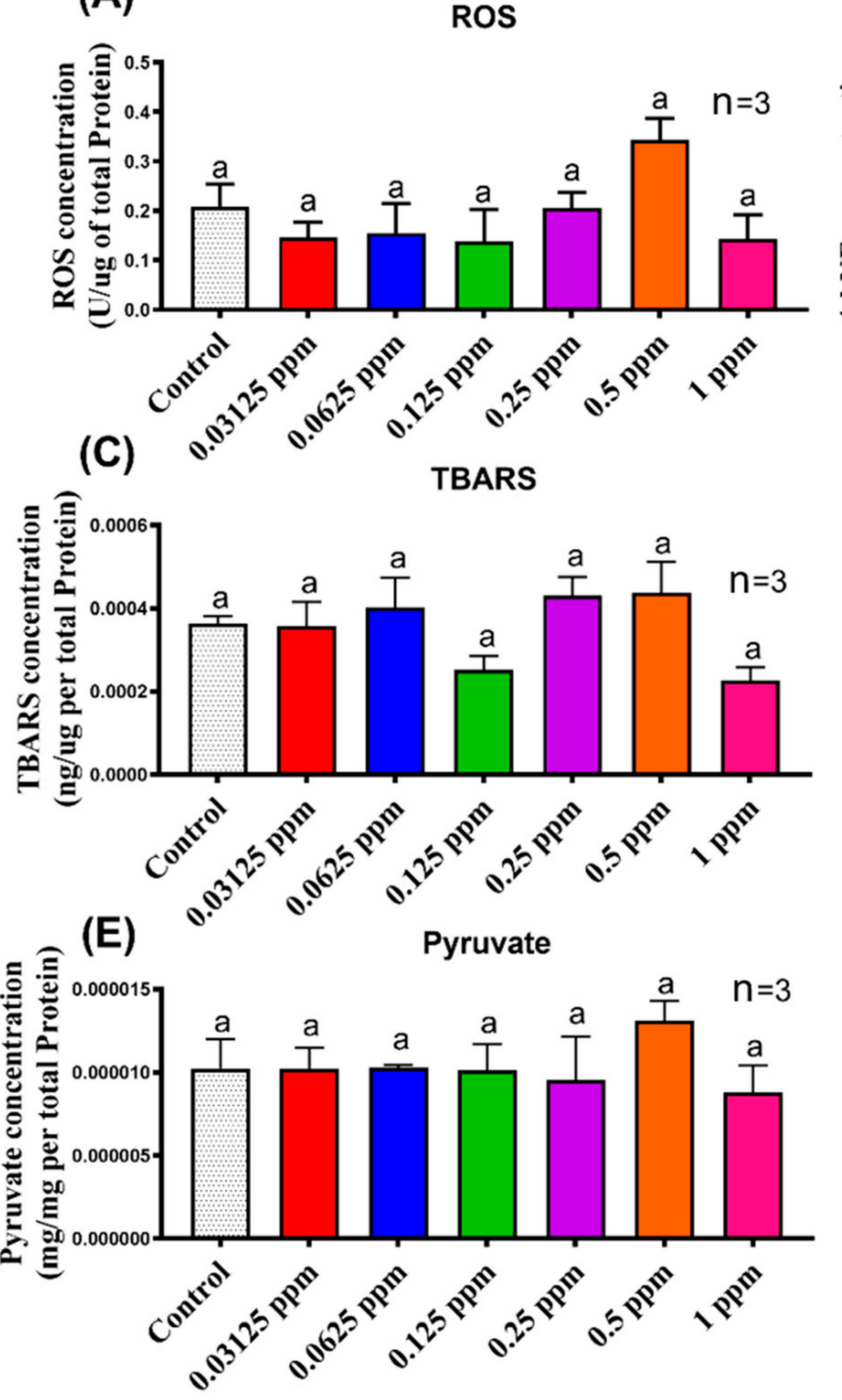

(B)
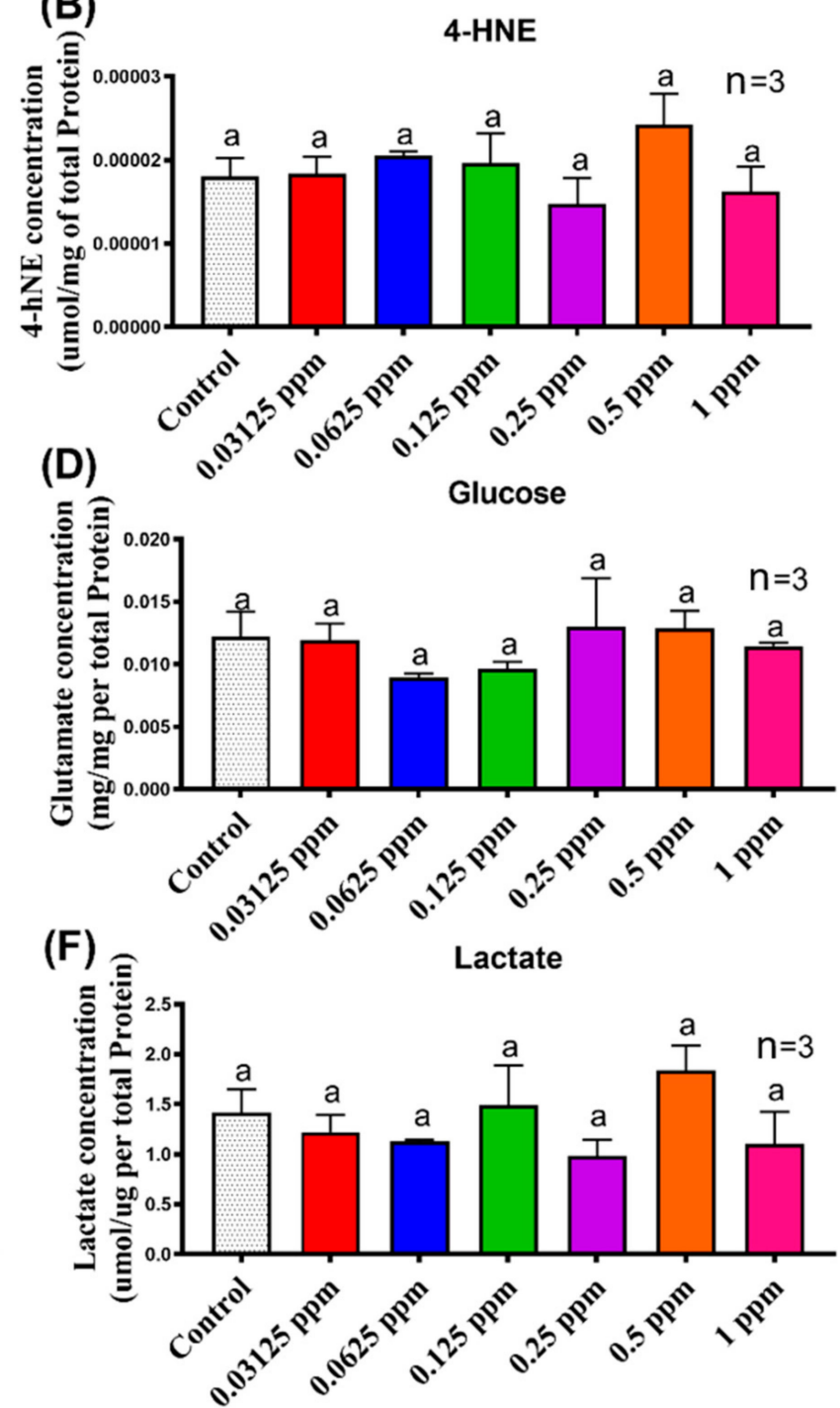

Figure 5. Evaluation of biomarker content in shrimp (Neocaridina denticulata) by enzyme-linked immunosorbent assay (ELISA) after exposure to different imidacloprid (IMI) concentrations. Here we used (A) ROS, (B) 4-HNE, and (C) TBARS to evaluate oxidative stress, (D) glucose, (E) pyruvate, and (F) lactate to evaluate energy metabolism. The data are expressed as mean with SEM. Statistical analysis was by one-way ANOVA test followed by Tukey post-hoc test (a is used as a label, different letter (a) above the error bars indicate a significant difference with $p<0.05$ ). 
(A)

\section{Total Distance Chronology}
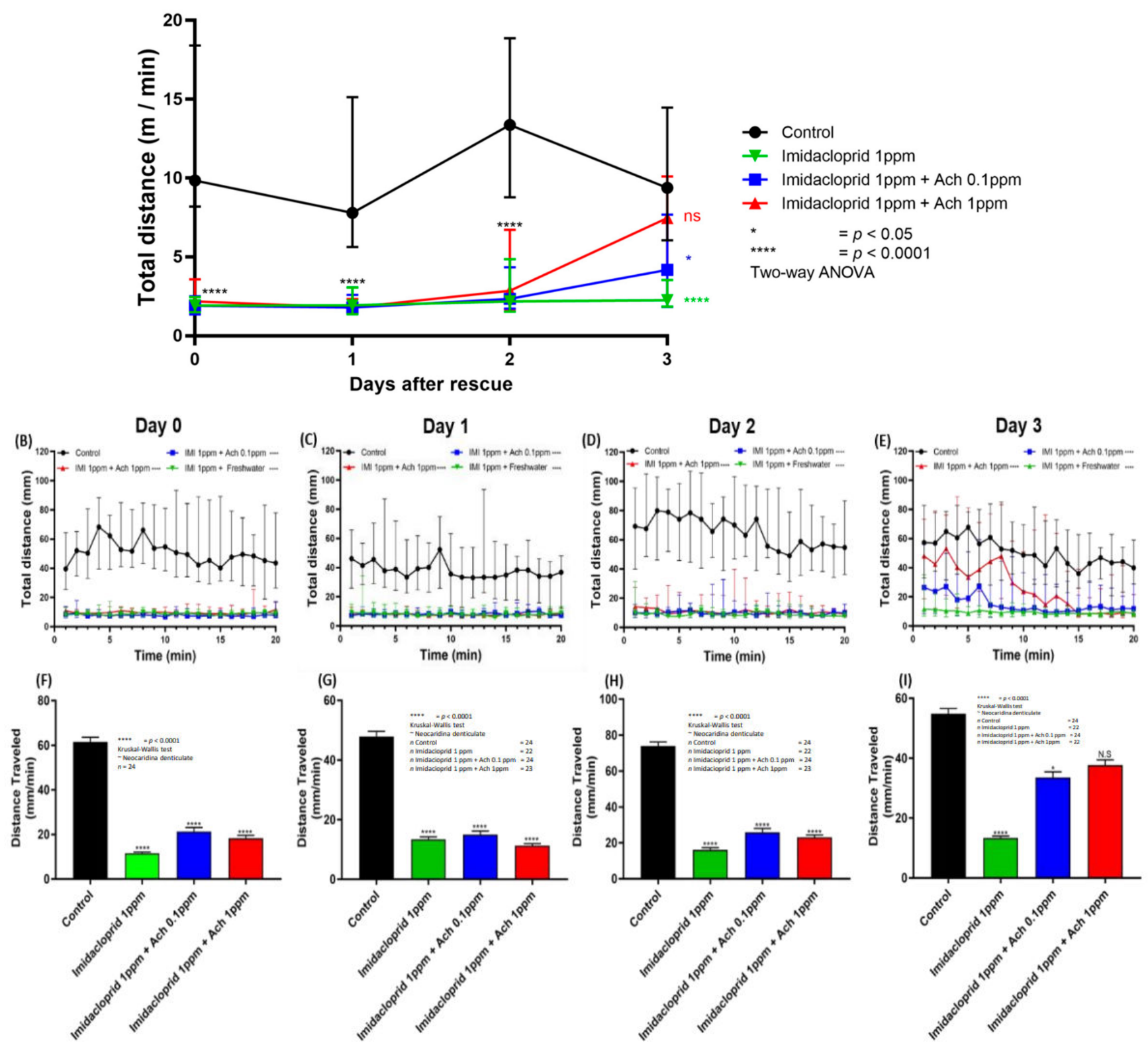

Figure 6. The rescue experiment showing acetylcholine can restore locomotor immobilization triggered by imidacloprid (IMI) in shrimp (Neocaridina denticulata). (A) Total distance chronology of shrimp treated with IMI and rescue using acetylcholine from days 0-3. (B) Total distance traveled chronology for $20 \mathrm{~min}$ locomotor activity test on day 0 of IMI treated shrimp. (C) Total distance traveled chronology of shrimp treated IMI rescue with acetylcholine for 20 min locomotor activity test on day 1. (D) Total distance traveled chronology of shrimp treated IMI rescue with acetylcholine for 20 min locomotor activity test on day 2. (E) Total distance traveled chronology of shrimp treated IMI rescue with acetylcholine for 20 min locomotor activity test on day 3. The data are expressed as mean with SEM and were analyzed using two-way ANOVA with Geisser-Greenhouse corrections. Dunnett's multiple comparison test for comparing all treatments with control was carried out to observe the main column (IMI) effect. (F) Total distance traveled locomotor activity test on day 0 of IMI treated shrimp. (G) Total distance traveled by shrimp treated IMI rescue with acetylcholine locomotor activity test on day 1. (H) Total distance traveled by shrimp treated IMI rescue with acetylcholine locomotor activity test on day 2. (I) Total distance traveled by shrimp treated IMI rescue with acetylcholine locomotor activity test on day 3. The data are expressed as mean with SEM and were analyzed by Kruskal-Wallis test along with Dunn's multiple comparisons test as follow-up test ( $n=24$ for all of the groups on day 0 , except 1 ppm IMI group on the day 1, 2, and 3 exposure time intervals $(n=22)$ ). $\left({ }^{*} p<0.05 ;{ }^{* * * *} p<0.0001\right)$. 


\subsection{Heartbeat and Gill Ventilation Rescue by Acetylcholine Administration}

Imidacloprid exposure on shrimp showed that it could affect the shrimp by reducing the heart and gill ventilation rate (Figure 3). We attempted to rescue the heart and gill ventilation rate of the exposed IMI-treated shrimp with acetylcholine from this result. The rescue experiment was started by exposing shrimp with IMI for $24 \mathrm{~h}$, and the heartbeat and gill ventilation rate were determined. After a day of exposure, IMI was removed from water in the shrimp tank. Water was replaced with acetylcholine with concentrations of $0.1 \mathrm{ppm}, 1 \mathrm{ppm}$, while the other contained clean water. Through days 1-3, the shrimp was exposed to acetylcholine and normal water after treatment with IMI. Based on our observations, IMI exposure to shrimp caused decreased heart and gill ventilation rates on day 0 compared to the control shrimp, which was not exposed to IMI (Figure 7A,B). Our rescue experiment results are consistent with a previous result illustrated in Figure 3C,E. These results proved that IMI could affect heart and gill ventilation rate in invertebrate organisms like N. denticulata. After a day of exposure to IMI, the shrimp were removed from the IMI solution environment and treated with acetylcholine to rescue heart and gill ventilation rates. The result on day 1 (Figure 7A) showed that exposure to acetylcholine 0.1 and $1 \mathrm{ppm}$ increases heartbeat, especially $1 \mathrm{ppm}$ acetylcholine, which showed a higher heartbeat percentage. The shrimp treated with clean water also showed an increment in heart rate percentage (Figure 7A). Meanwhile, gill ventilation rates were reduced even after acetylcholine exposure on day 1 . All the groups treated with acetylcholine at test concentrations of $0.1 \mathrm{ppm}, 1 \mathrm{ppm}$ along with the clean water showed reduced gill ventilation rates on day 1 (Figure 7B). For heart rate measurements starting at day 1 , the increment already has been noted, and finally, on day 3 , the heart rate in all the groups is already under similar levels with the control groups. Based on this result, we conclude that removal of IMI and exposure to acetylcholine could rescue the shrimp's heart rate (Figure 7A). Furthermore, acetylcholine seems to work differently on gill ventilation rates. While heart rate has been successfully increased, gill ventilation is decreased significantly on day 1 . With 1-ppm acetylcholine exposure, gill ventilation was increased on day 2 and decreased on day 3 (Figure 7B). This indicates that acetylcholine could affect heartbeat while it works differently on gill ventilation rate by decreasing it. From this result, we want to prove our hypothesis by exposing the shrimp directly to acetylcholine and check the effect.

Acetylcholine exposures at 0.1 and $1 \mathrm{ppm}$ concentrations seemed to have similar effects as we found in the rescue experiment. Shrimp treated with 0.1 and 1 ppm acetylcholine showed higher heart rates than a control shrimp. After exposure to acetylcholine starting from day 0 , the heart rate level was already higher than the control group. After exposure in 2 days, both acetylcholine-treated groups showed a significantly higher heartbeat percentage than the control groups. On day 3 of acetylcholine exposure, the shrimp showed higher heart rates than on day 2 exposure (Figure 7C). From this experiment, we were able to prove that acetylcholine exposure to shrimp can boost heartbeat rate. Compared to gill ventilation, the results showed an opposite trend compared to the heartbeat experiment. It seemed that acetylcholine works differently in gill ventilation because both low and high acetylcholine concentration exposure showed a decreased level of the gill ventilation rate (Figure 7D). 

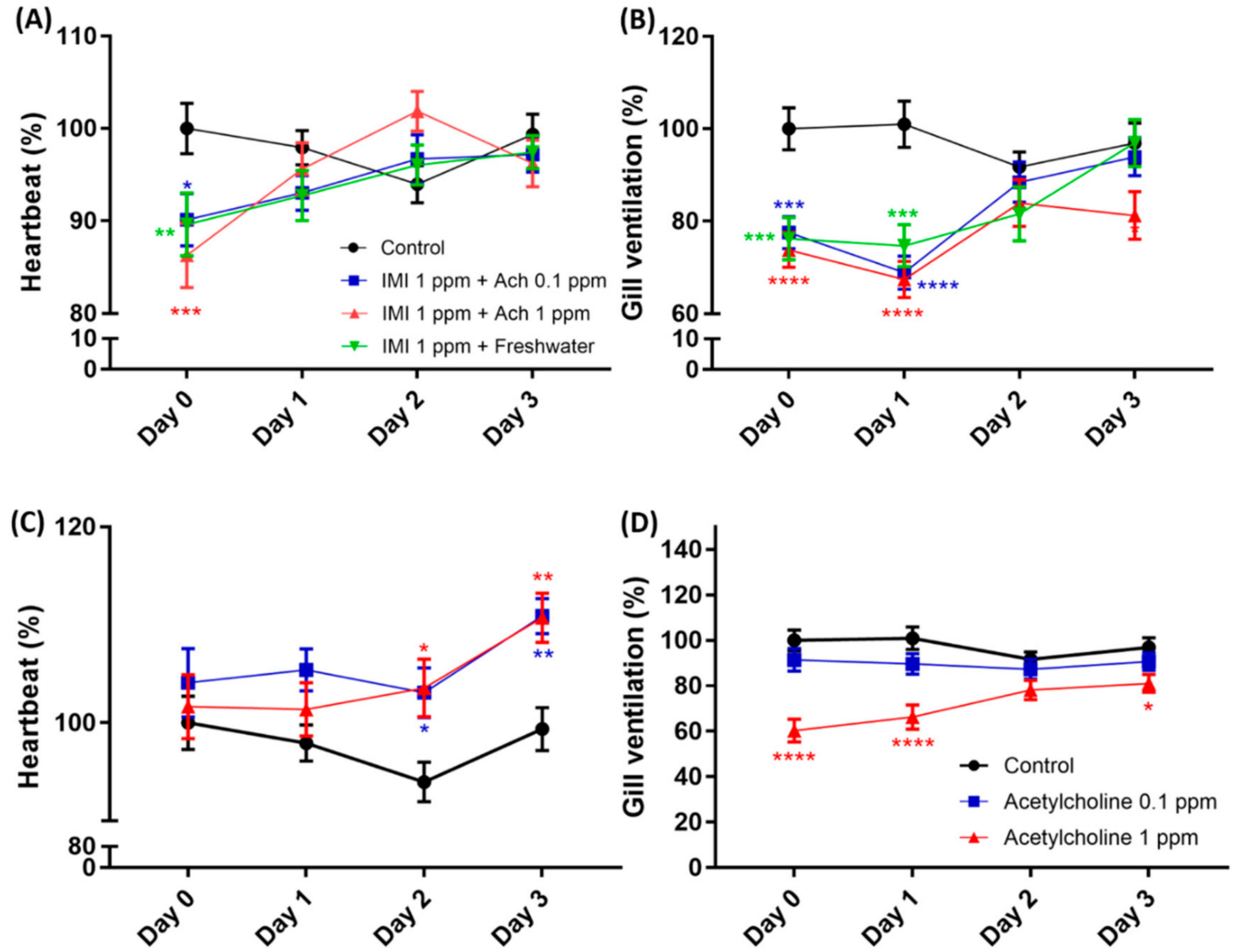

Figure 7. The rescue experiment showing acetylcholine can restore the heartbeat and gill ventilation beating in shrimp (Neocaridina denticulata) after being treated with imidacloprid (IMI). (A) The heart rate of shrimp after exposure to IMI for $24 \mathrm{~h}$ (day 0 ) and rescue by acetylcholine exposure through days 1-3. (B) Gill ventilation rate of shrimp after exposure to IMI for $24 \mathrm{~h}$ (day 0 ) and rescue by acetylcholine through days 1-3. The data are expressed as mean with SEM and were analyzed using two-way ANOVA along with Dunnet's multiple comparisons as a follow-up test $(n=24$ for the control group on day 0 through $3 ; n=24$ for 1 ppm IMI rescue with 0.1 ppm acetylcholine group on days $0-2$, except for day 3 $(n=23) ; n=24$ for 1 ppm IMI rescue with 1 ppm acetylcholine group on day 0 , on day $1-2 n=23$, while on day $3 n=22$; $n=22$ for $1 \mathrm{ppm}$ IMI rescue with fresh water on day 0 , while from day 1 through day $3 n=21$ ). (C) Heart rate of shrimp after exposure to acetylcholine for 3 days (Control positive). (D) Gill ventilation rate of shrimp after exposure to IMI for 3 days (Control positive). The data are expressed as mean with SEM and were analyzed by two-way ANOVA along with Dunnet's multiple comparisons as a follow-up test $(n=24$ for the control group on day 0 through $3 ; n=24$ for 0.1 ppm acetylcholine on day $0-2$, except for day $3(n=23) ; n=24$ for 1 ppm acetylcholine group on day $0, n=23$ on day 1 , while $n=22$ on days $2-3) .\left({ }^{*} p<0.05{ }^{* *} p<0.01 ;{ }^{* * *} p<0.001\right.$; $\left.^{* * * *} p<0.0001\right)$.

\subsection{Molecular Docking}

In probing and visualizing imidacloprid and acetylcholine's binding mechanisms to nicotinic acetylcholine receptors, molecular docking studies were carried out. Nicotinic acetylcholine receptors (nAChRs) are ligand-gated ion channels mediating excitatory cholinergic neurotransmission in vertebrates and invertebrates [45,46]. Each $\mathrm{nAChR}$ is a pentamer that assembles as five subunits. Acetylcholine binds to extracellular ligandbinding domains at the subunit interfaces, which is formed by loops A-F. Upon binding acetylcholine and agonists, the central cation-permeable ion channel opens transiently, propagating nerve impulses.

Due to the shortage of public information for shrimp nAChR crystal structure, acetylcholine binding protein (AChBP) from the snail Lymnaea stagnalis is considered a surrogate marker of the ligand-binding domain in $\mathrm{nAChRs} \mathrm{for} \mathrm{loops} \mathrm{A-F,} \mathrm{which} \mathrm{are} \mathrm{highly} \mathrm{conserved}$ and was used for our in silico experiments [28]. The simulation results of molecular docking show that the binding energy of acetylcholine binding protein to IMI is $-6.0 \mathrm{kcal} / \mathrm{mol}$. 
In contrast, the binding energy of acetylcholine is $-4.2 \mathrm{kcal} / \mathrm{mol}$ to the nAChR. The intermolecular interactions of the atoms $<2.5$ angstroms from IMI and acetylcholine can be visualized in the docking poses seen in Figure 8A,B, respectively. The relatively strong binding was mainly observed through conventional hydrogen bonds between the nitrogen of the pyridine moiety in IMI with Trp143 and amidine $N$-nitro with the phenolic functionality of Tyr192. Other significant interactions include a pi-sigma bond between the pyridine and hydroxyl alkyl of Thr144 and multiple pi-alkyl bonds of the methylpyridine with amino acid residues Arg104, Leu112, Tyr113, and Met114 (Figure 8A). On the other hand, acetylcholine interacted only via weak van der Waals with Trp143 of nAChR (Figure 8B).

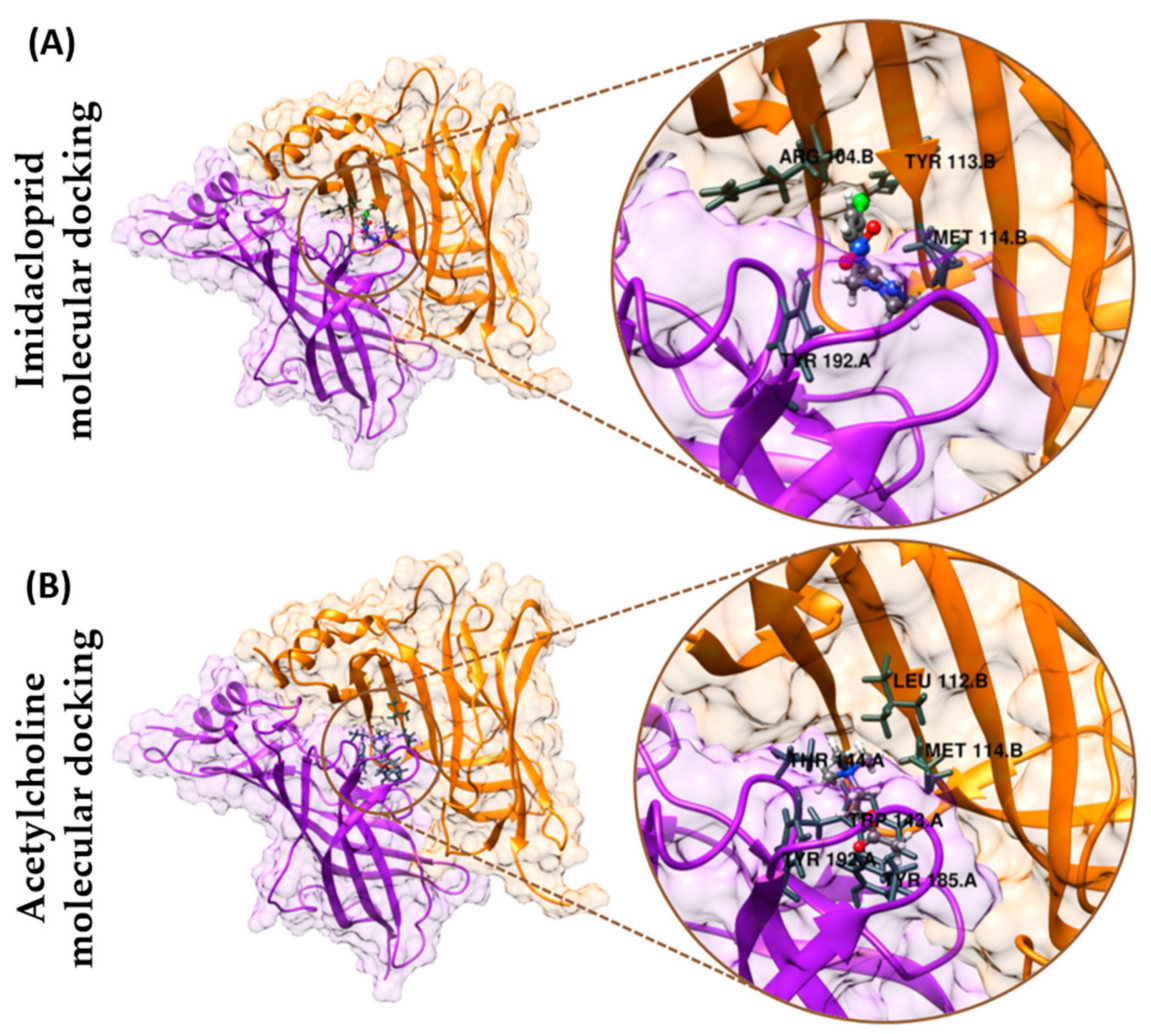

Figure 8. Molecular docking experiment to compare the binding affinity of imidacloprid (IMI) and acetylcholine to nicotinic acetylcholine receptor (nAChR). (A) The docking pose includes chains A (purple) and B (orange), representing two of the five subunits that make nAChR. The binding of IMI is seen in the interface of the two chains. IMI, at $<2.5$ angstroms, exhibited intermolecular interactions with Tyr192 of chain A and Arg104, Tyr113, Met114 of chain B. A wide-angle view (left) and a focused image (right) are illustrated. (B) The binding of acetylcholine is seen in the chain A (purple) and B (orange) interface. At $<2.5$ angstroms, acetylcholine exhibited intermolecular interactions with Trp143, Thr144, Tyr185, Tyr192 of chain A and Leu112, Met114 of chain B. A wide-angle view (left) and a focused image (right) are illustrated.

\section{Discussion}

Our study highlighted freshwater shrimp N. denticulata as an excellent laboratory animal model for a specific practice, such as ecotoxicology. This species is a decapod (crustacean) that is widely available in the aquarium industry as pets, aquatic plant cleaners, and fish bait. They are well-known to rapidly spread and expand their population in freshwater ecosystems [47]. N. denticulata has much potential to become a crustacean ecotoxicological model. Their characteristics are very much applicable to a wide variety of purposes and meet laboratory study criteria. The adult's body length can grow to around $2.85 \mathrm{~cm}$, making them experimentally simple trackable animals. The tissue-specific toxicity of insecticide can be addressed by conducting experimental validation at either 
biochemical or molecular levels in isolated tissues. They have also easily grown species that can tolerate a wide range of $\mathrm{pH}(6.5-8.0)$ and temperature $\left(22-25^{\circ} \mathrm{C}\right.$, up to $\left.30^{\circ} \mathrm{C}\right)$. These animals can be easily maintained in small freshwater tanks at room temperature with simple filtration and aeration for survival and reproduction [48]. Female $N$. denticulata is also highly reproductive, producing 20-30 fertilized eggs in 30 days post-mating. This makes them readily available and accessible to culture in the laboratory. Additionally, the draft genome [48] and transcriptome [49-52] of N. denticulata have been decoded, making them an ideal model for functional genomic studies [53]. This study investigated $N$. denticulata as an ideal aquatic nontarget invertebrate model for pesticide toxicity analysis. Given this scenario, studies to explore IMI physiologic effects in shrimp N. denticulata, a crustacean widely distributed throughout Taiwan [54], represent a relevant effort.

Imidacloprid is a member of the neonicotinoid class of insecticides [55]. It acts on the postsynaptic nicotinic acetylcholine receptors (nAChRs) located in the central nervous system of insects [56]. It functions as an agonist to the five-subunit arranged ligand-gated ion channel causing a biphasic response (initial increase in spontaneous discharge frequency followed by a complete block to nerve propagation) [57]. Its usefulness as an insecticide comes from its ability to selectively bind to nAChRs of insects primarily due to the insect's differential sensitivity and vertebrate nAChR subtypes. However, its widespread use sparked concerns over other nontarget organisms [58]. Previous studies prove that IMI has a selective affinity to nAChR in insects' nervous tissues $[18,19]$. Other studies also prove the opposite result where nontarget organisms can also be affected by IMI. Several studies have proven IMI's ability to interact with nAChRs of the mouse brain, thus causing toxicity to mice [20]. This experiment showed that the bioactivation of IMI contributes to its toxicity to mammals [20]. Several studies also demonstrated that IMI exposure affects various fish species. For example, IMI was shown to be toxic in Japanese medaka larvae. This insecticide could induce developmental retardation and severely hamper the fitness of the fish [59]. Another study showed that IMI exposure of Prochilodus lineatus leads to DNA damage and oxidative stress in different tissues [60]. Another experiment also showed that acute IMI toxicity could cause inflammation and oxidative stress in common carps [61]. These previous studies proved that IMI might have adverse and toxic effects on nontarget organisms. Due to low soil binding and high-water solubility of neonicotinoids, aquatic ecosystems are at high risk of contamination, mainly due to run-off events. It is important to be aware of the adverse effect of IMI in aquatic organisms such as shrimp. Simultaneous studies of cardiac, gill ventilation, and behavioral effects induced by IMI are not well explored in any aquatic toxicity model, despite its wide use in agriculture and structural similarities with several neurotoxic drugs including nicotine and chlorpyrifos.

In this study, among the three assayed endpoints, we noted that shrimp locomotion activity could become the most sensitive parameter for chemical toxicity evaluation in terms of heartbeat, gill ventilation, and locomotion. Starting from a low dose at the ppb level, the locomotor activity already significantly decreased (Figure 2). Meanwhile, other parameters such as heartbeat and gill ventilation displayed significant alterations only at high dose IMI treatment at ppm levels. This result is consistent with our previous findings in zebrafish larvae and Daphnia neonates [62], showing locomotor activity alteration as a sensitive marker to conduct toxicological screening. Our study also corroborates a previous study in brown shrimp (Farfantepenaeus aztecus), showing behavior changes noticeably after IMI exposure. After exposure to IMI, the affected brown shrimp could not swim normally, stopped moving, and sprawled at the bottom of the tank, but their legs moved involuntarily and died [63]. We also observed similar behavioral alterations in freshwater shrimp N. denticulata after IMI exposure showing that at the lowest concentration of IMI $(0.03125 \mathrm{ppm})$, paralysis-like symptoms have already been triggered. Another experiment conducted in aquatic insect Simulium vittatum also strengthened our result, where IMI treated insects showed abnormal behavior and muscle controls [64]. Other experiments conducted in zebrafish also showed IMI exposure resulting in neurobehavioral defects [65]. Reduced locomotion activity may be caused by IMI interaction with nAChR, thus prevent- 
ing acetylcholine from binding with $\mathrm{nAChR}$ and causing dysregulation of the nervous system [20-22]. Dysregulation can lead to decreased locomotion activity. Paralysis also can be a reason why there is reduced locomotion activity [63]. After exhibiting abnormal swimming behaviors, shrimp legs will stop moving and become paralyzed. This leads shrimp to hunger and death [63]. Some of our treated shrimp also showed identical behaviors, and finally, some of them died at the final part of the test. The inability of treated shrimp to move and find any food can cause the shrimp to die. However, IMI may directly affect shrimp locomotor, inability to swim or moving the legs, and paralysis that primarily caused death in shrimp treated with IMI.

Another test we did was the cardiac rhythm for the shrimp. We noted that shrimp heartbeat and gill ventilation movement showed a decline when either lack of food and nutrition or IMI exposure. Previous studies in Mytilus edulis showed starvation to reduce the frequency of heartbeat and the rate of oxygen uptakes. After feeding, the frequency of heartbeat increased gradually to active levels [66]. In addition to starvation, some factors can affect the heartbeat such as environmental factors like temperature. A previous study has also proven that temperature can also affect the heartbeat. In Daphnia, heart rates display a good positive relationship with ambient water temperature [67]. In M. edulis, a high temperature was observed to increase the heartbeat. However, it will be reduced again after reaching a certain degree, and a lower temperature will reduce the heartbeat [66]. Interestingly, we found a consistent environmental condition to keep shrimp as an important factor in maintaining data reproducibility. Meanwhile, in the IMI treated group, we found that even at the lowest concentration, it could reduce heartbeat until the same level as shrimp starvation (Figure 3C). The highest concentration of IMI lowered heartbeat compared to the control and starvation group (Figure 3C). IMI exposure is known to cause a depressed heart rate. Previous experiments demonstrated that IMI exposure to $D$. magna could affect heartbeat by reducing the heart rate of $D$. magna in all of the tested concentrations [67]. In another D. magna study, a similar result was noted when D. magna was exposed to neonicotinoid insecticides. IMI, as neonicotinoid, has similar mechanisms and could affect $D$. magna by reducing heart rates after $48 \mathrm{~h}$ of exposure [68]. Our study disclosed similar results with other studies where $N$. denticulata with IMI caused a reduction in heart rate and gill ventilation compared to the control (Figure 3C,E). Another evidence that can prove our result is a study investigating the effects of exposing zebrafish to IMI where reduced heartbeat, blood flow, and malformation were observed [69]. As an invertebrate animal model in this study, shrimp has also demonstrated similar results after incubation with IMI. Our study proved that IMI indeed could attack and affect nontarget organisms like freshwater shrimp.

The reduction in locomotion activity, heart rate, and gill ventilation might also be linked with energy metabolism and oxidative stress. Hence, we investigated lipid peroxidation biomarkers such as ROS, TBARS, and 4-HNE to check whether oxidative stress is responsible for locomotion immobilization in $N$. denticulata after IMI exposure. Evidence collected from biochemical assays did not favor our previous hypothesis since IMI acute treatment had no adverse effect on energy metabolism and oxidative stress (Figure 5). Therefore, we proposed that reduced locomotion, heart rate, and gill ventilation are not influenced by biomarker alterations but are primarily contributed by acetylcholine binding blockage to the nAChRs due to IMI interference. The study from in vivo results was confirmed with in silico molecular docking, which revealed a higher binding affinity of IMI towards nAChR than acetylcholine (Figure 8). This result supports our hypothesis that there may be an IMI mechanism underlying in affected shrimp locomotion activity by binding with $\mathrm{nAChR}$, thus preventing acetylcholine from interacting with $\mathrm{nAChR}$. Our study supports a conserved mechanism of action between $N$. denticulata and other insect counterparts, showing superior binding affinity of IMI with nAChR compared to acetylcholine, thus impairing locomotion, heart rate, and maxilliped movements.

Since the molecular docking result revealed that IMI exhibits a stronger binding affinity to $\mathrm{nAChR}$ than acetylcholine, our hypothesis of acetylcholine overdosing might 
lead to competitive binding to nAChR. Thus, a rescue experiment was performed. IMI may remain on the water surface for a long time due to high water solubility [70]. IMI cannot be hydrolyzed by ACh esterase, causing interruption to nAChR leading to long-lasting effects, such as loss of reflexes, muscular weakness, and paralysis [71]. Consequently, it alters invertebrate movements and leads to starvation and death. A previous study revealed that IMI toxicity to freshwater amphipod Gammarus pulex could recover relatively fast after transferring to clean water [72]. However, our study found that locomotor immobilization triggered by IMI exposure could not be easily restored after transferring to clean water (Figure 6A). The heartbeat and gill ventilation rescue experiment also showed similar results with the locomotion. Even after moving IMI treated shrimp to fresh, clean water, the biological effect is maintained even after 3 days (Figure 8A,B). However, when acetylcholine was administered at either 0.1 or $1 \mathrm{ppm}$, the nontarget animals can recover from IMI sublethal effects, even though acetylcholine treatment needs 2 days to recover from IMI exposure. Meanwhile, for heartbeat and gill ventilation rescue experiments, acetylcholine could rescue the heartbeat faster compared to locomotion (Figure 7A). The heartbeat rescue experiment is supported by other results where acetylcholine-treated shrimp showed higher heartrate compared to the control (Figure 7C). The rescue experiment showed that exogenous acetylcholine exposure could compete with IMI to nACRs and restore the adverse effects triggered by IMI. Our results are consistent with a prior study conducted in insects showing IMI can be dissociated from $\mathrm{nAChR}$ by acetylcholine and other nicotinic ligands epibatidine, $\alpha$-bungarotoxin, and methyllycaconitine [73]. Therefore, our findings support IMI reversible high binding affinity to $\mathrm{nAChR}$. The rescue effect of acetylcholine needs quite some time to be showcased. The reason is that IMI is relatively stable and only slowly hydrolyzes in neutral water. Photodegradation of IMI in natural sunlight is also weak, making IMI in normal water persist for a long time [74]. Locomotion showed a rescue effect during the last day, while the heartbeat rate showed a rescue effect on day 2. The persistence of IMI in water seems to be why they still can bind with nAChR thus blocking acetylcholine as a natural neurotransmitter (the mechanism was summarized in Figure 9).

\section{Imidacloprid exposure}

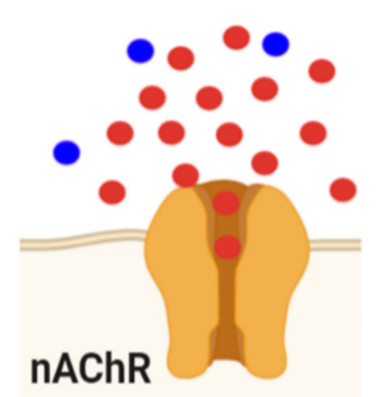

Acetylcholine rescue

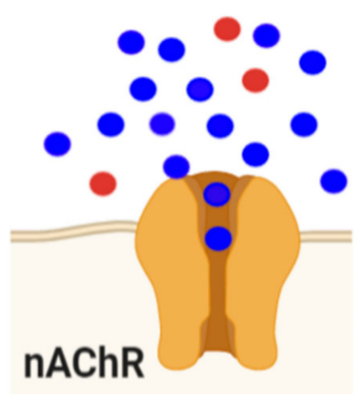

$\begin{array}{ccc}\downarrow & \text { Locomotor activity } \\ \downarrow & \uparrow \\ \downarrow & \text { Gill ventilation rate } & \uparrow \\ \text { Heartbeat rate } & \uparrow\end{array}$

Figure 9. Model to explain why imidacloprid (highlighted by red color) can trigger locomotor compromise in shrimp and has been rescued by acetylcholine (highlighted by blue color) administration. Exogenous imidacloprid has high affinity to nicotinic acetylcholine receptor (nAChR) and compete with endogenous acetylcholine. The $\mathrm{nAChR}$ blockage by imidacloprid leads to signal transduction blockage and reduces locomotor activity, gill ventilation and heartbeat rates. However, when the shrimp has been exposed to excess amount to exogenous acetylcholine, those physiological compromises can be rescued. 
Finally, we successfully demonstrated that IMI treatment for $24 \mathrm{~h}$ could lead to immobilization, reduce heart rate, decrease gill ventilation, and ultimately cause death in shrimp. This insecticide's adverse effect is specific to its target and could pose harmful effects to nontarget organisms like the shrimp $N$. denticulata. Butcherine et al. reported that neonicotinoid exposure might raise adverse effects on wild shrimp fisheries and aquaculture productivity due to adverse effects on feeding and lipid contents in tiger shrimp [75]. Bardran et al. also reported that IMI exposure at corresponding EPA benchmark concentrations could sharply reduce survival rates and delay molting in juvenile brown shrimp (Farfantepenaeus aztecus) [63]. All these reports support our study that showcases IMI adverse effects on $N$. denticulata in locomotion and cardiac rhythm.

\section{Conclusions}

We successfully created and demonstrated the imidacloprid adverse effect to nontarget organism such as freshwater shrimp ( $N$. denticulata). Shrimp exposed to imidacloprid insecticide showed immobilization, reduce heart rate, decrease gill ventilation, and ultimately could cause death. This insecticide proven to be toxic not only to target organism, but also harmful to non-target organism such as freshwater shrimp. According to this experiment, the use of chemical such as insecticide that could harm environment should be regulated with caution. The use of imidacloprid in the future should pay attention to our environment. We also provide possible mechanism to explain imidacloprid could trigger immobilization with rescue experiment. In this consideration, our finding opens a new avenue to perform translational studies beneficial to shrimp fisheries and aquaculture industries. In situations where cultured shrimp with high economic value been polluted by IMI, the possibility of conducting acetylcholine rescue to prevent significant economic loss is useful and relevant.

Supplementary Materials: The following are available online at https:/ /www.mdpi.com/2076-392 $1 / 10 / 3 / 391 /$ s1. Video S1, Comparison of the locomotor activity alterations in shrimp after 3 days imidacloprid exposure (video is speed up $40 \times$ ). Video S2, The heartbeat and gill ventilation in a control shrimp. Video S3, The heartbeat and gill ventilation in shrimp after 3 days imidacloprid exposure.

Author Contributions: Conceptualization, P.S., C.-D.H. and H.-T.L.; methodology and software, M.E.S., K.H.-C.C., J.-C.H., H.-M.C., F.S. (Fiorency Santoso), A.H., B.T.N.H., K.A.K. and R.A.F.; validation, F.S. (Ferry Saputra), G.A. and M.J.M.R.; formal analysis and investigation, P.S., M.E.S. and G.A.; writing - original draft preparation, P.S., A.P.G.M., C.-D.H. and H.-T.L.; supervision, C.-D.H. and H.-T.L.; project administration and funding acquisition, C.-D.H. and H.-T.L. All authors have read and agreed to the published version of the manuscript.

Funding: This study was supported by the grants sponsored by the Ministry of Science Technology (MOST107-2622-B-033-001-CC2 and MOST108-2622-B-033-001-CC2) to C.-D.H. and MOST107-2321B415-001 to H.-T.L. The funders have no role in study design, data collection and analysis, decision to publish, or manuscript preparation.

Institutional Review Board Statement: Ethical review and approval were waived for this study, due to non-vertebrate model been conducted.

Informed Consent Statement: Not applicable.

Data Availability Statement: Original data and videos can be obtained from authors upon request.

Acknowledgments: We thank the Freshwater Bioresource Center at National Chiayi University for providing freshwater shrimp.

Conflicts of Interest: The authors declare no conflict of interest. The funders had no role in the design of the study; in the collection, analyses, or interpretation of data; in the writing of the manuscript, or in the decision to publish the results. 


\section{Appendix A}

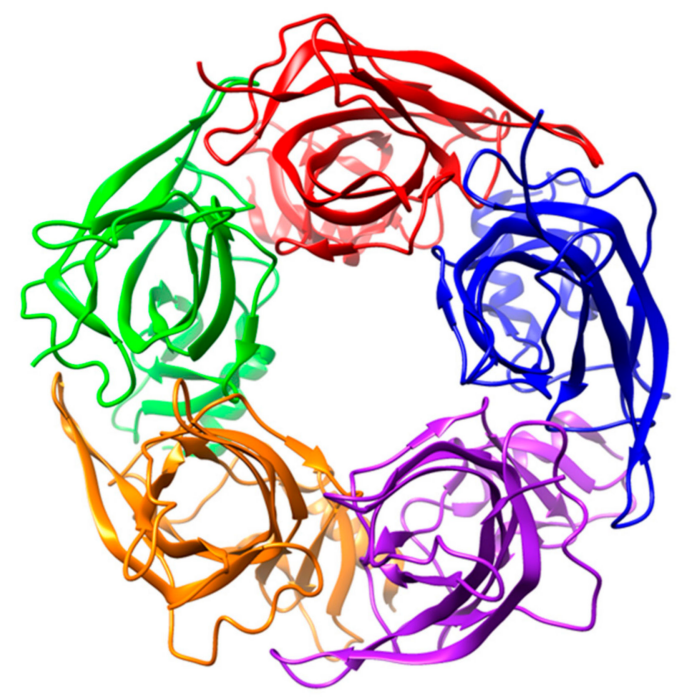

Figure A1. The homopentameric structure of the acetylcholine binding protein (AChBP) from the snail Lymnaea stagnalis; retrieved from the Protein Data Bank.

\section{References}

1. Chaudhuri, K.; Selvaraj, S.; Pal, A. Studies on the genotoxicity of endosulfan in bacterial systems. Mutat. Res. Genet. Toxicol. Environ. Mutagenesis 1999, 439, 63-67. [CrossRef]

2. Salameh, P.; Brochard, P. Respiratory symptoms in children and exposure to pesticides. Eur. Respir. J. 2003, 22, 507-512. [CrossRef]

3. Paolini, M.; Sapone, A.; Gonzalez, F.J. Parkinson's disease, pesticides and individual vulnerability. Trends Pharmacol. Sci. 2004, 25, 124-129. [CrossRef] [PubMed]

4. Flower, K.B.; Hoppin, J.A.; Lynch, C.F.; Blair, A.; Knott, C.; Shore, D.L.; Sandler, D.P. Cancer risk and parental pesticide application in children of Agricultural Health Study participants. Environ. Health Perspect. 2004, 112, 631-635. [CrossRef]

5. Kumar, S. Occupational exposure associated with reproductive dysfunction. J. Occup. Health 2004, 46, 1-19. [CrossRef]

6. Hook, S.E.; Doan, H.; Gonzago, D.; Musson, D.; Du, G.; Kookana, R.; Sellars, M.J.; Kummar, A. The impacts of modern-use pesticides on shrimp aquaculture: An assessment for north eastern Australia. Ecotoxicol. Environ. Saf. 2018, 148, 770-780. [CrossRef]

7. Yan, S.H.; Wang, J.H.; Zhu, L.S.; Chen, A.M. Thiamethoxam induces oxidative stress and antioxidant response in zebrafish (Danio rerio) livers. Environ. Toxicol. 2016, 31, 2006-2015. [CrossRef]

8. Azevedo-Pereira, H.; Lemos, M.; Soares, A.M. Effects of imidacloprid exposure on Chironomus riparius Meigen larvae: Linking acetylcholinesterase activity to behaviour. Ecotoxicol. Environ. Saf. 2011, 74, 1210-1215. [CrossRef] [PubMed]

9. Jemec, A.; Tisler, T.; Drobne, D.; Sepcić, K.; Fournier, D.; Trebse, P. Comparative toxicity of imidacloprid, of its commercial liquid formulation and of diazinon to a non-target arthropod, the microcrustacean Daphnia magna. Chemosphere 2007, 68, 1408-1418. [CrossRef] [PubMed]

10. Stoughton, S.J.; Liber, K.; Culp, J.; Cessna, A. Acute and chronic toxicity of imidacloprid to the aquatic invertebrates Chironomus tentans and Hyalella azteca under constant-and pulse-exposure conditions. Arch. Environ. Contam. Toxicol. 2008, 54, 662-673. [CrossRef]

11. Dani, J.A.; De Biasi, M. Cellular mechanisms of nicotine addiction. Pharmacol. Biochem. Behav. 2001, 70, 439-446. [CrossRef]

12. Matsuda, K.; Buckingham, S.D.; Freeman, J.C.; Squire, M.D.; Baylis, H.A.; Sattelle, D.B. Effects of the $\alpha$ subunit on imidacloprid sensitivity of recombinant nicotinic acetylcholine receptors. Br. J. Pharmacol. 1998, 123, 518-524. [CrossRef]

13. Matsuda, K.; Buckingham, S.D.; Kleier, D.; Rauh, J.J.; Grauso, M.; Sattelle, D.B. Neonicotinoids: Insecticides acting on insect nicotinic acetylcholine receptors. Trends Pharmacol. Sci. 2001, 22, 573-580. [CrossRef]

14. Tomizawa, M.; Casida, J.E. Neonicotinoid insecticide toxicology: Mechanisms of selective action. Annu. Rev. Pharm. Toxicol 2005, 45, 247-268. [CrossRef]

15. Huang, D.-J.; Chen, H.-C. Effects of chlordane and lindane on testosterone and vitellogenin levels in green neon shrimp (Neocaridina denticulata). Int. J. Toxicol. 2004, 23, 91-95. [CrossRef]

16. Sung, H.-H.; Lin, Y.-H.; Hsiao, C.-Y. Differential immune responses of the green neon shrimp (Neocaridina denticulate) to dipropyl phthalate. Fish Shellfish Immunol. 2011, 31, 511-515. [CrossRef] [PubMed]

17. Jeschke, P.; Nauen, R.; Schindler, M.; Elbert, A. Overview of the status and global strategy for neonicotinoids. J. Agric. Food Chem. 2011, 59, 2897-2908. [CrossRef] 
18. Simon-Delso, N.; Amaral-Rogers, V.; Belzunces, L.P.; Bonmatin, J.M.; Chagnon, M.; Downs, C.; Furlan, L.; Gibbons, D.W.; Giorio, C.; Girolami, V.; et al. Systemic insecticides (neonicotinoids and fipronil): Trends, uses, mode of action and metabolites. Environ. Sci. Pollut. Res. 2015, 22, 5-34. [CrossRef]

19. Sanchez-Bayo, F. Insecticides mode of action in relation to their toxicity to non-target organisms. J. Environ. Anal. Toxicol. 2012, 21, 973-992.

20. Chao, S.L.; Casida, J.E. Interaction of imidacloprid metabolites and analogs with the nicotinic acetylcholine receptor of mouse brain in relation to toxicity. Pestic. Biochem. Physiol. 1997, 58, 77-88. [CrossRef]

21. Shimomura, M.; Yokota, M.; Ihara, M.; Akamatsu, M.; Sattelle, D.B.; Matsuda, K. Role in the selectivity of neonicotinoids of insect-specific basic residues in loop D of the nicotinic acetylcholine receptor agonist binding site. Mol. Pharmacol. 2006, 70, 1255-1263. [CrossRef]

22. Barrantes, F. Structural basis for lipid modulation of nicotinic acetylcholine receptor function. Brain Res. Rev. 2004, 47, 71-95. [CrossRef]

23. Tiwari, P.; Dwivedi, S.; Singh, M.P.; Mishra, R.; Chandy, A. Basic and modern concepts on cholinergic receptor: A review. Asian Pac. J. Trop. Dis. 2013, 3, 413-420. [CrossRef]

24. Beers, W.; Reich, E. Structure and activity of acetylcholine. Nature 1970, 228, 917-922. [CrossRef] [PubMed]

25. Trott, O.; Olson, A.J. AutoDock Vina: Improving the speed and accuracy of docking with a new scoring function, efficient optimization, and multithreading. J. Comput. Chem. 2010, 31, 455-461. [CrossRef] [PubMed]

26. Landis, W.; Sofield, R.; Yu, M.-H.; Landis, W.G. Introduction to Environmental Toxicology: Impacts of Chemicals upon Ecological Systems; CRC Press: Boca Raton, FL, USA, 2003.

27. Sampurna, B.P.; Audira, G.; Juniardi, S.; Lai, Y.-H.; Hsiao, C.-D. A simple imagej-based method to measure cardiac rhythm in zebrafish embryos. Inventions 2018, 3, 21. [CrossRef]

28. Ihara, M.; Okajima, T.; Yamashita, A.; Oda, T.; Hirata, K.; Nishiwaki, H.; Morimoto, T.; Akamatsu, M.; Ashikawa, Y.; Kuroda, S.I. Crystal structures of Lymnaea stagnalis AChBP in complex with neonicotinoid insecticides imidacloprid and clothianidin. Invertebr. Neurosci 2008, 8, 71-81. [CrossRef] [PubMed]

29. Pettersen, E.F.; Goddard, T.D.; Huang, C.C.; Couch, G.S.; Greenblatt, D.M.; Meng, E.C.; Ferrin, T.E. UCSF Chimera-A visualization system for exploratory research and analysis. J. Comput. Chem 2004, 25, 1605-1612. [CrossRef]

30. Hanwell, M.D.; Curtis, D.E.; Lonie, D.C.; Vandermeersch, T.; Zurek, E.; Hutchison, G.R. Avogadro: An advanced semantic chemical editor, visualization, and analysis platform. J. Cheminform. 2012, 4, 17. [CrossRef] [PubMed]

31. Wang, J.; Wang, W.; Kollman, P.A.; Case, D.A. Automatic atom type and bond type perception in molecular mechanical calculations. J. Mol. Graph. Model. 2006, 25, 247-260. [CrossRef]

32. Yang, J.; Roy, A.; Zhang, Y.J.B. Protein-ligand binding site recognition using complementary binding-specific substructure comparison and sequence profile alignment. Bioinformatics 2013, 29, 2588-2595. [CrossRef] [PubMed]

33. Yamanaka, O.; Takeuchi, R. UMATracker: An intuitive image-based tracking platform. J. Exp. Biol. 2018, 221. [CrossRef]

34. Kim, S.-K.; Guo, Q.; Jang, I.-K. Effect of biofloc on the survival and growth of the postlarvae of three penaeids (Litopenaeus vannamei, Fenneropenaeus chinensis, and Marsupenaeus japonicus) and their biofloc feeding efficiencies, as related to the morphological structure of the third maxilliped. J. Crustacean Biol. 2015, 35, 41-50.

35. Estes, P.S. Cardiovascular and Respiratory Responses of the Ghost Shrimp, Callianassa Californiensis Dana, to the Pesticide Carbaryl and Its Hydrolytic Product 1-Naphthol. Master's Thesis, Oregon State University, Corvallis, OR, USA, 1986.

36. Olima, C.; Pablo, F.; Lim, R. Comparative tolerance of three populations of the freshwater shrimp (Paratya australiensis) to the organophosphate pesticide, chlorpyrifos. Bull. Env.. Contam. Toxicol 1997, 59, 321-328. [CrossRef]

37. Alexander, C.; Hindley, J.; Jones, S. Structure and function of the third maxillipeds of the banana prawn Penaeus merguiensis. Mar. Biol. 1980, 58, 245-249. [CrossRef]

38. Hsiao, C.-D.; Wu, H.-H.; Malhotra, N.; Liu, Y.-C.; Wu, Y.-H.; Lin, Y.-N.; Saputra, F.; Santoso, F.; Chen, K.H.-C. Expression and Purification of Recombinant GHK Tripeptides Are Able to Protect against Acute Cardiotoxicity from Exposure to WaterborneCopper in Zebrafish. Biomolecules 2020, 10, 1202. [CrossRef]

39. Guzik, P.; Piskorski, J.; Krauze, T.; Wykretowicz, A. Heart rate asymmetry by Poincaré plots of RR intervals. Biomed. Tech. 2006, 51, 272-275. [CrossRef]

40. Piskorski, J.; Guzik, P. Geometry of the Poincaré plot of RR intervals and its asymmetry in healthy adults. Physiol. Meas. 2007, 28, 287. [CrossRef]

41. Slaninova, A.; Smutna, M.; Modra, H.; Svobodova, Z. REVIEWS Oxidative stress in fish induced by pesticides. Neuroendocr. Lett 2009, 30, 2.

42. Martelli, F.; Zhongyuan, Z.; Wang, J.; Wong, C.-O.; Karagas, N.E.; Roessner, U.; Rupasinghe, T.; Venkatachalam, K.; Perry, T.; Bellen, H.J. Low doses of the neonicotinoid insecticide imidacloprid induce ROS triggering neurological and metabolic impairments in Drosophila. Proc. Natl. Acad. Sci. USA 2020, 117, 25840-25850. [CrossRef]

43. Tsai, M.-C.; Huang, T.-L. Thiobarbituric acid reactive substances (TBARS) is a state biomarker of oxidative stress in bipolar patients in a manic phase. J. Affect. Disord. 2015, 173, 22-26. [CrossRef] [PubMed]

44. Kim, D.H.; Kwack, S.J.; Yoon, K.S.; Choi, J.S.; Lee, B.-M. 4-Hydroxynonenal: A superior oxidative biomarker compared to malondialdehyde and carbonyl content induced by carbon tetrachloride in rats. J. Toxicol. Environ. Healthpart A 2015, 78, 1051-1062. [CrossRef] 
45. Liu, M.-Y.; Casida, J.E. High affinity binding of $[3 \mathrm{H}]$ imidacloprid in the insect acetylcholine receptor. Pestic. Biochem. Physiol. 1993, 46, 40-46. [CrossRef]

46. McGehee, D.S.; Role, L.W. Physiological diversity of nicotinic acetylcholine receptors expressed by vertebrate neurons. Annu. Rev. Physiol. 1995, 57, 521-546. [CrossRef]

47. Levitt-Barmats, Y.A.; Yanai, Z.; Cohen, T.M.; Shenkar, N. Life-history traits and ecological characteristics of the ornamental shrimp Neocaridina denticulata (De Haan, 1844), recently introduced into the freshwater systems of Israel. Aquat. Invasions 2019, 14, 684-702. [CrossRef]

48. Kenny, N.J.; Sin, Y.W.; Shen, X.; Zhe, Q.; Wang, W.; Chan, T.F.; Tobe, S.S.; Shimeld, S.M.; Chu, K.H.; Hui, J.H. Genomic sequence and experimental tractability of a new decapod shrimp model, Neocaridina denticulata. Mar. Drugs 2014, 12, 1419-1437. [CrossRef] [PubMed]

49. Huang, C.-W.; Chu, P.-Y.; Wu, Y.-F.; Chan, W.-R.; Wang, Y.-H. Identification of Functional SSR Markers in Freshwater Ornamental Shrimps Neocaridina denticulata Using Transcriptome Sequencing. Mar. Biotechnol. 2020, 22, 772-785. [CrossRef] [PubMed]

50. Kim, N.; Jeon, E.; Jung, S.; Ahn, S.; Park, M.; Seo, J. Gene expression profiling and expression analysis of freshwater shrimp (Neocaridina denticulata denticulata) using expressed sequence tags and short-term exposure to copper. J. Environ. Biol. 2018, 39, 51-57. [CrossRef]

51. Xing, K.; Liu, Y.; Yan, C.; Zhou, Y.; Sun, Y.; Su, N.; Yang, F.; Xie, S.; Zhang, J. Transcriptome analysis of Neocaridina denticulate sinensis under copper exposure. Gene 2020, 764, 145098. [CrossRef]

52. Chou, C.-H.; Huang, H.-Y.; Huang, W.-C.; Hsu, S.-D.; Hsiao, C.-D.; Liu, C.-Y.; Chen, Y.-H.; Liu, Y.-C.; Huang, W.-Y.; Lee, M.-L. The aquatic animals' transcriptome resource for comparative functional analysis. BMC Genom. 2018, 19, 161-170. [CrossRef]

53. Mykles, D.L.; Hui, J.H. Neocaridina denticulata: A decapod crustacean model for functional genomics. Integr. Comp. Biol. 2015, 55, 891-897. [CrossRef]

54. Chen, Y.; Zang, L.; Liu, M.; Zhang, C.; Shen, G.; Du, W.; Sun, Z.; Fei, J.; Yang, L.; Wang, Y. Ecological risk assessment of the increasing use of the neonicotinoid insecticides along the east coast of China. Environ. Int 2019, 127, 550-557. [CrossRef]

55. Pittendrigh, B.R.; Margam, V.M.; Sun, L.; Huesing, J.E. Resistance in the postgenomics age. Insect Resist. Manag. Biol. Econ. Predict. 2008, 2, 39-68.

56. Dalefield, R. Veterinary Toxicology for Australia and New Zealand; Elsevier: Amsterdam, The Netherlands, 2017; Volume 1. Available online: https: / / www.elsevier.com/books/veterinary-toxicology-for-australia-and-new-zealand/dalefield/978-0-12-420227-6 (accessed on 21 January 2021).

57. Gupta, R.C. Biomarkers in Toxicology; Academic Press: Cambridge, MA, USA, 2019.

58. Miles, J.C.; Hua, J.; Sepulveda, M.S.; Krupke, C.H.; Hoverman, J.T. Effects of clothianidin on aquatic communities: Evaluating the impacts of lethal and sublethal exposure to neonicotinoids. PLoS ONE 2017, 12, e0174171. [CrossRef]

59. Vignet, C.; Cappello, T.; Fu, Q.; Lajoie, K.; De Marco, G.; Clérandeau, C.; Mottaz, H.; Maisano, M.; Hollender, J.; Schirmer, K. Imidacloprid induces adverse effects on fish early life stages that are more severe in Japanese medaka (Oryzias latipes) than in zebrafish (Danio rerio). Chemosphere 2019, 225, 470-478. [CrossRef]

60. Vieira, C.E.D.; Pérez, M.R.; Acayaba, R.D.A.; Raimundo, C.C.M.; dos Reis Martinez, C.B. DNA damage and oxidative stress induced by imidacloprid exposure in different tissues of the Neotropical fish Prochilodus lineatus. Chemosphere 2018, 195, 125-134. [CrossRef]

61. Özdemir, S.; Altun, S.; Arslan, H. Imidacloprid exposure cause the histopathological changes, activation of TNF- $\alpha$, iNOS, 8-OHdG biomarkers, and alteration of caspase 3, iNOS, CYP1A, MT1 gene expression levels in common carp (Cyprinus carpio L.). Toxicol. Rep. 2018, 5, 125-133.

62. Hussain, A.; Audira, G.; Malhotra, N.; Uapipatanakul, B.; Chen, J.-R.; Lai, Y.-H.; Huang, J.-C.; Chen, K.H.-C.; Lai, H.-T.; Hsiao, C.-D. Multiple Screening of Pesticides Toxicity in Zebrafish and Daphnia Based on Locomotor Activity Alterations. Biomolecules 2020, 10, 1224. [CrossRef]

63. Al-Badran, A.A.; Fujiwara, M.; Mora, M.A. Effects of insecticides, fipronil and imidacloprid, on the growth, survival, and behavior of brown shrimp Farfantepenaeus aztecus. PLoS ONE 2019, 14, e0223641. [CrossRef]

64. Overmyer, J.; Mason, B.; Armbrust, K. Acute toxicity of imidacloprid and fipronil to a nontarget aquatic insect, Simulium vittatum Zetterstedt cytospecies IS-7. Bull. Environ. Contam. Toxicol. 2005, 74, 872-879. [CrossRef]

65. Crosby, E.B.; Bailey, J.M.; Oliveri, A.N.; Levin, E.D. Neurobehavioral impairments caused by developmental imidacloprid exposure in zebrafish. Neurotoxicol. Teratol. 2015, 49, 81-90. [CrossRef]

66. Widdows, J. Effect of temperature and food on the heart beat, ventilation rate and oxygen uptake of Mytilus edulis. Mar. Biol. 1973, 20, 269-276. [CrossRef]

67. Santoso, F.; Krylov, V.V.; Castillo, A.L.; Saputra, F.; Chen, H.-M.; Lai, H.-T.; Hsiao, C.-D. Cardiovascular Performance Measurement in Water Fleas by Utilizing High-Speed Videography and ImageJ Software and Its Application for Pesticide Toxicity Assessment. Animals 2020, 10, 1587. [CrossRef]

68. Bownik, A.; Pawłocik, M.; Sokołowska, N. Effects of neonicotinoid insecticide acetamiprid on swimming velocity, heart rate and thoracic limb movement of Daphnia magna. Pol. J. Nat. Sci. 2017, 32, 481-493.

69. Tišler, T.; Jemec, A.; Mozetič, B.; Trebše, P. Hazard identification of imidacloprid to aquatic environment. Chemosphere 2009, 76, 907-914. [CrossRef] 
70. Guo, L.; Dai, Z.; Guo, J.; Yang, W.; Ge, F.; Dai, Y. Oligotrophic bacterium Hymenobacter latericoloratus CGMCC 16346 degrades the neonicotinoid imidacloprid in surface water. Amb Express 2020, 10, 1-13. [CrossRef] [PubMed]

71. Wu, Y.-Y.; Luo, Q.-H.; Hou, C.-S.; Wang, Q.; Dai, P.-L.; Gao, J.; Liu, Y.-J.; Diao, Q.-Y. Sublethal effects of imidacloprid on targeting muscle and ribosomal protein related genes in the honey bee Apis mellifera L. Sci. Rep. 2017, 7, 1-8. [CrossRef]

72. Nyman, A.-M.; Hintermeister, A.; Schirmer, K.; Ashauer, R. The insecticide imidacloprid causes mortality of the freshwater amphipod Gammarus pulex by interfering with feeding behavior. PLoS ONE 2013, 8, e62472. [CrossRef]

73. Lind, R.J.; Clough, M.S.; Reynolds, S.E.; Earley, F.G. [3H] Imidacloprid Labels High-and Low-Affinity Nicotinic Acetylcholine Receptor-like Binding Sites in the AphidMyzus persicae (Hemiptera: Aphididae). Pestic. Biochem. Physiol. 1998, 62, 3-14. [CrossRef]

74. Liu, W.; Zheng, W.; Ma, Y.; Liu, K.K. Sorption and degradation of imidacloprid in soil and water. J. Environ. Sci. Health Part B 2006, 41, 623-634. [CrossRef]

75. Butcherine, P.; Kelaher, B.P.; Taylor, M.D.; Barkla, B.J.; Benkendorff, K. Impact of imidacloprid on the nutritional quality of adult black tiger shrimp (Penaeus monodon). Ecotoxicol. Environ. Saf. 2020, 198, 110682. [CrossRef] [PubMed] 